\title{
Character Strengths Profiles in Medical Professionals and Their Impact on Well-Being
}

\author{
Alexandra Huber ${ }^{1}$, Cornelia Strecker ${ }^{2}$, Timo Kachel2,3, Thomas Höge ${ }^{2}$ and Stefan Höfer ${ }^{1 *}$ \\ ${ }^{1}$ Department of Medical Psychology, Medical University of Innsbruck, Innsbruck, Austria, ${ }^{2}$ Institute of Psychology, University \\ of Innsbruck, Innsbruck, Austria, ${ }^{3}$ Department of Psychiatry, Psychotherapy and Psychosomatics, University Hospital of \\ Psychiatry II, Medical University of Innsbruck, Innsbruck, Austria
}

\section{OPEN ACCESS}

Edited by: Philippe Dubreuil, Université du Québec à Trois-Rivières,

Canada

Reviewed by:

Benjamin Dreer,

University of Erfurt, Germany

Maria Christina Meyers,

Tilburg University, Netherlands

*Correspondence: Stefan Höfer

stefan.hoefer@i-med.ac.at

Specialty section:

This article was submitted to Personality and Social Psychology, a section of the journal

Frontiers in Psychology

Received: 28 May 2020 Accepted: 04 December 2020 Published: 23 December 2020

Citation:

Huber A, Strecker C, Kachel T, Höge T and Höfer S (2020) Character

Strengths Profiles in Medical Professionals and Their Impact on Well-Being

Front. Psychol. 11:566728. doi: 10.3389/fpsyg.2020.566728
Character strengths profiles in the specific setting of medical professionals are widely unchartered territory. This paper focused on an overview of character strengths profiles of medical professionals (medical students and physicians) based on literature research and available empirical data illustrating their impact on well-being and work engagement. A literature research was conducted and the majority of peer-reviewed considered articles dealt with theoretical or conceptually driven 'virtues' associated with medical specialties or questions of ethics in patient care (e.g., professionalism, or what makes a good physician). The virtues of compassion, courage, altruism, and benevolence were described most often. Only a limited number of papers addressed character strengths of medical students or physicians according to the VIA-classification. Those articles showed that the VIA-character strengths fairness, honesty, kindness, and teamwork were considered most often by respondents to be particularly important for the medical profession. Available cross-sectional (time span: six years) and longitudinal (time span: three years) data regarding VIA-character strengths profiles of medical professionals were analyzed ( $N=584$ medical students, 274 physicians). These profiles were quite homogenous among both groups. The character strengths fairness, honesty, judgment, kindness, and love had the highest means in both samples. Noteworthy differences appeared when comparing medical specialties, in particular concerning general surgeons and psychiatrists, with the former reporting clearly higher levels of e.g., honesty $(d=1.02$ ) or prudence $(d=1.19)$. Long-term results revealed significant positive effects of character strengths on well-being and work engagement (e.g., perseverance on physicians' work engagement) but also significant negative effects (e.g., appreciation of beauty and excellence on students' well-being). Further, hope was significantly associated both positively with physicians' well-being and negatively with students' work engagement, possibly indicating specific issues concerning medical education or hospital working conditions. According to the modern-day physician's pledge, medical professionals should pay attention to their own well-being and health. Therefore, promoting self-awareness and character building among medical professionals could be a beneficial strategy.

Keywords: character strengths profiles, VIA-classification, medical students, physicians, well-being, work engagement 


\section{INTRODUCTION}

Character strengths are inherent in all humans. They are reflected in everyday thoughts, attitudes, and behaviors, and have a positive relation to one's well-being (Peterson and Seligman, 2004). The discipline of Positive Psychology (Seligman and Csikszentmihalyi, 2000) defines character strengths as a group of positively valued and moral traits that an individual can possess, enabling growth, flourishing and moral excellence (Seligman, 2002). The 'Values in Action' (VIA) classification describes 24 character strengths, assigned to six virtues (courage, humanity, justice, temperance, transcendence, wisdom) that have been theoretically considered as being important for over 3000 years across different religions, cultures, and traditions (Peterson and Seligman, 2004). Seligman (2002) suggests that individuals ought to utilize their character strengths by enforcing them according to their life circumstances to obtain well-being and to increase positive benefits. Some character strengths have been identified to be more strongly related with life satisfaction and occupational well-being than others, the so-called 'happiness strengths' curiosity, gratitude, hope, love, and zest (e.g., Peterson and Park, 2006; Buschor et al., 2013; Littman-Ovadia et al., 2016). In another study, perseverance and social intelligence were most strongly associated with life satisfaction (beside hope, love, and zest) and humor playing an important role for wellbeing (Martínez-Martí and Ruch, 2014). Applying e.g., gratitude (Emmons and McCullough, 2003; Machado et al., 2019) or kindness (Otake et al., 2006) led to higher levels of well-being, and generally the application of character strengths at work was related to various positive experiences (e.g., pleasure, work engagement, meaning) and job satisfaction (Littman-Ovadia and Steger, 2010; Seligman, 2011; Harzer and Ruch, 2012, 2013). Others identified appreciation of beauty and excellence, creativity, judgment, love of learning, and humility to be least related with life satisfaction (Park et al., 2004).

Furthermore, specific character strengths might be more prevalent among certain groups of people or professions than among others. Such 'profiles' might exist because (1) a certain job rather attracts people with a certain distinct set of character strengths, and (2) shared environments (e.g., study or working conditions, occupational and organizational structures, processes and cultures, trainers/colleagues as role models, etc.) shape individual character strengths in a similar vein toward a 'collective profile' (Peterson and Seligman, 2004). Particularly medical students and hospital physicians need to master comprehensive demands and strains. Additionally, they often report impaired well-being or even mental illness raising the question on possible underlying character strengths profiles. Medical students reported more depressive symptoms and higher levels of distress with regard to their health compared to the general United States population (Dyrbye et al., 2014), impaired mental health (e.g., Brazeau et al., 2014) and well-being (e.g., Dunn et al., 2008), or early onset of burnout symptoms (Kachel et al., 2020a). Moreover, origins of recurrent physician burnout were identified with studies showing a prevalence of $45 \%$ up to $70 \%$ to have these symptoms during medical education at least once (Dyrbye et al., 2008; Ishak et al., 2013) entailing health impairing consequences (e.g., Jackson et al., 2016). Physicians are further confronted with various work demands and job strains (e.g., workload, time pressure, emotional labor, social stressors, cognitive demands; Angerer and Weigl, 2015) and when they feel unwell, the performance of health-care systems as well as patient care can be impaired (e.g., Wallace et al., 2009; Klein et al., 2010). Compared to the general population, an increased burnout risk was reported (resident physicians: 60\%; physicians: 51\%; Dyrbye et al., 2014), and in addition depression, substance abuse and suicide occurred above-average (Gold et al., 2013). Indeed, medical students and hospital physicians are exposed to challenging circumstances but just therefore, actively pleading for individual positive experiences in terms of applying one's character strengths could be particularly beneficial for their well-being and health (e.g., Hershberger, 2005).

Therefore, this study aimed to determine possible character strengths profiles of medical professionals based on a focused literature research on medical students' and physicians' virtues and VIA-character strengths and own empirical data. Possible differences regarding character strengths profiles of various sub-groups (e.g., age, sex, different medical specialties) will be discussed as well as their respective relevance and relation to medical professionals' well-being and work engagement.

\section{VIRTUES AND CHARACTER STRENGTHS IN THE LITERATURE}

The science of psychology as it has been practiced until the 1980s/90s needed to be enriched by focusing more on positive aspects of human experiences and behavior (Peterson and Seligman, 2004) as the historically developed imbalance toward studying psychopathology and negative aspects within this discipline (e.g., mental disorders, diagnoses, and treatment; Cassell, 2002; Sheldon and Lyubomirsky, 2004; Harzer and Ruch, 2013) threatened to turn unilateral. Thus, a paradigm shift was heralded by Positive Psychology in the late 1990s by Martin E. P. Seligman as one of its founders. Based on the fundamental virtues of courage, humanity, justice, temperance, transcendence, and wisdom, character strengths were emphasized again (Peterson and Seligman, 2004). These strengths are considered quite stable characteristics of an individual, therefore a theoretical overlap with personality traits is possible (Park et al., 2004; Peterson and Seligman, 2004). However, one conceptual difference between character strengths and personality traits is their normative vs. descriptive perspective on individual differences. Character strengths are positively valued (normative) whereas personality traits are usually described in an unbiased way on continuums (e.g., Five-Factor Model = Big Five; McCrae and Costa, 1997). Only pathological personality aspects were always clearly negatively valued (e.g., Dimensional Assessment of Personality Pathology; Livesley, 2006). The positive, moral valuation of 'character' led to its exclusion from personality psychology in the 1930s as Gordon Allport defined that 'Character is personality evaluated, and personality is character devaluated. Since character is an unnecessary concept for psychology, the term will not appear 
again in this volume...' (c.f. Linley et al., 2007). Today's research is questioning this exclusion as there is new evidence indicating that virtues, and therefore character strengths respectively, are an expression of personality rather than 'moral reasoning and cognitive development' (see Cawley et al., 2000). For example, people scoring high on the Big Five dimension 'agreeableness' (= being friendly and compassionate vs. challenging/callous) also reported higher levels of forgiveness, gratitude, hope, kindness, prudence or self-regulation (Haslam et al., 2004; Brose et al., 2005; Wood et al., 2008), while 'neuroticism' (= being sensitive and nervous vs. resilient/confident) negatively predicted bravery and hope (Macdonald et al., 2008). The latter study tested a theoretically derived model relating the six VIA-virtues to the Big Five revealing no correlate for the VIA-virtue of transcendence. Thus, it appears evident that character strengths and personality traits overlap but are not redundant, also adding incremental validity in, for instance, predicting life satisfaction (Park et al., 2004; West, 2006).

Moreover, occupational preferences and choices can also be ascribed to other sorts of dispositions than virtues and character strengths (e.g., interests, abilities, skills). For example, studies using the 'Strong-Campbell Vocational Interest Inventory' (Campbell, 1977) or being based on the 'RIASEC typology of careers' (realistic - investigative - artistic - social - enterprising - conventional; Holland, 1997) have investigated occupational preferences, medical careers, and chosen specialties. One study identified all medical disciplines to be throughout investigativesocial' (Borges et al., 2004), whereas another study by Petrides and McManus (2004) revealed that e.g., surgery is rather a 'realistic' discipline (including people who like to work with things: here hands and tools, needing high levels of technical proficiency, craftsmanship and practical skills), internal medicine can be more assigned to the 'investigative' category (including people who like to work with data: exploring symptoms and relating them to latent causes to make a diagnosis), and psychiatry was considered to be more 'artistic' (including people who like to work with ideas: interpreting patients' problems using various bio-psycho-social theories and responding individually to each patient). In turn, physicians who selected specialties with more pronounced social features also had higher scores on the Big Five dimension of 'agreeableness', whereas higher 'neuroticism' implied rather a preference for 'artistic' and an aversion for 'realistic' or 'enterprising' specialties (Woods et al., 2016). Overall, two meta-analyses found three moderate relationships between personality traits (Big Five) and vocational interests (RIASEC) of medical students (see Duffy et al., 2009): 'extraversion' with 'enterprising' and 'social', and 'openness to experience' with 'artistic.' However, character strengths as positively valued aspects of personality have hardly been related to the medical vocation before. In summary, the principle idea is that awareness of one's individual character strengths may increase well-being and positivity, promote self-awareness on possible career paths, and improve workplace productivity and relationships. Nonetheless, there might be an accumulation of specific character strengths within certain professions, like 'typical' character strengths due to common life circumstances, experiences, study conditions or job specifications.
In the following, an overview of the most important findings of the conducted literature research will be presented. The searching strategy included the following terms: 'character strengths' or 'values in action' or 'virtues' and 'medical students' or instead of the students 'medical doctors' or 'physicians' or 'resident physicians.' The literature research was conducted in the following databases: APA Psycinfo, APA Psycarticles, Psyndex, Web of Science (Core Collection), Socindex, Pubmed/Medline, and Eric. In total, 160 hits revealed for medical students (time frame: 1971-2020) and 626 for physicians (time frame: 18162020). After screening all results, matching the search key with regards to content and considering double hits as well as multiple articles reporting on the same data, 43 peer-reviewed papers for medical students and 81 for physicians remained.

\section{Medical Students}

Relating to virtues of medical students in general, most findings referred to (achieving) professionalism, virtuous caring, and good physicianhood. All these qualities overlap with Edmund Pellegrino's proposed fundamental virtues of the medical profession, namely benevolence, courage, compassion, fidelity to trust, intellectual honesty, and truthfulness (Pellegrino, 2002). This prominent bioethicist pled for their tuition in medical school from the very beginning alongside knowledge and skills (Jacobson et al., 2006; Buyx et al., 2008; O'Sullivan and Toohey, 2008; Wear and Zarconi, 2008; Behrens and Fellingham, 2014; Magalhães-Sant'Ana, 2015; Shepherd et al., 2018). Therefore, the 'Accreditation Council for Graduate Medical Education' embedded respective virtues into graduate medical education in the late 1990s (overview in Larkin et al., 2005) as teaching professionalism and developing a good character can be understood as educators' responsibility (Sehiralti et al., 2010; Carey et al., 2015). The 'explicit' professionalism curriculum puts patients into the center and supports altruistic attitudes, but the 'implicit' or 'hidden' curriculum that is defined by the learning environment in which it takes place (Hafferty and Franks, 1994) is often contrary, e.g., educators teaching opposite values by valuing appearance, formality, and conformity wrongly as 'professional' (Brainard and Brislen, 2007; Karches and Sulmasy, 2016). However, medical students' altruistic behavior and empathy seem to be susceptible (Schweller et al., 2017; Sanjai and Gopichandran, 2018) and should be fostered by respective early curricular interventions during medical education.

Relating to character strengths of medical students in terms of the VIA-classification (Peterson and Seligman, 2004), eight empirical studies could be identified. Five studies derived from the 'WELL-MED' project (see section 'Participants and Procedure' for details) with two focusing on the applicability of character strengths and their associations with healthrelated outcomes (Hausler et al., 2017a; Huber et al., 2020), one illuminating the correlations of character strengths and different well-being aspects (Hausler et al., 2017b), one examining the development of cynicism (Kachel et al., 2020a), and one validating the VIA-120 short form (Höfer et al., 2019). However, none of these studies focused on identifying a certain profile of medical students. Thus, empirical data from this study will pursue this question. The top five character strengths of the medical 
students from this German-speaking sample were fairness, honesty, judgment, kindness, and love. In total, 19 character strengths met the criteria for at least a slight possession (see Harzer and Ruch, 2013). When asking British medical students to identify and rank the VIA-character strengths that they think best represent (a) their personal character and what they think (b) a good doctor would need, the most frequently answers were: (a) fairness, honesty, kindness, perseverance and teamwork, and (b) fairness, honesty, judgment, kindness, leadership and teamwork (Kotzee et al., 2017). In another study (Jones, 2013), British final year medical students were asked: 'What are the most important character strengths of a good doctor?'. This study revealed honesty as the leading character strength, followed by teamwork, judgment, and kindness (descending order), whereas other frequently selected character strengths like love of learning, perseverance, or social intelligence were considered less important. Final year medical students in Oman rated as well (in descending order) honesty, teamwork, and judgment as being the most important VIA-character strengths for a physician, followed by fairness, kindness, and love of learning (Panambur et al., 2017). These six VIA-character strengths were also identified by them as most commonly observed in their teachers during the patient encounter. However, except for the 'WELL-MED' studies, participating medical students did not complete the VIAquestionnaire themselves revealing their own character strengths but they ranked the 24 character strengths from a descriptive list, respectively.

\section{Physicians}

Most research on physicians' virtues referred to professionalism accompanied with being a good doctor (also against cultural and/or spiritual backgrounds) and certain role virtues depending on medical specialty. Virtues have been already discussed in the early Stoic philosophy (e.g., Zeno's four cardinal virtues: bravery, justice, temperance, and wisdom; Papadimos, 2004) and found their way into medical ethics through John Gregory (17241773) proposing compassion, integrity, self-effacement, and selfsacrifice to be essential for professionalism (Chervenak and McCullough, 2004). Modern clinical medicine and physicianpatient relationships were significantly influenced by the book 'The Virtues in Medical Practice' by Pellegrino and Thomasa (1993; as cited in Fuks et al., 2012; Olivieri, 2018) addressing again the fundamental virtues (cf. above in 'medical students'). Summarizing historical and modern literature, some virtues recur. In particular, compassion was discussed oftentimes to play a central role (e.g., Lopez and Dyck, 2009; Gelhaus, 2012; Aramesh, 2017) as well as courage (e.g., Shelp, 1984; Fugelli, 1999; Begley, 2008), altruism (e.g., Bishop and Rees, 2007), humility (e.g., Coulehan, 2011; DuBois et al., 2013), hope (e.g., Bryan, 2007; Miller, 2012), and practical wisdom (e.g., Corcoran et al., 2016; Bain, 2018). Professionalism in other countries or cultures is partially focusing on other values like in Korea, where physicians evaluated duties (e.g., responsibility, veracity) to be of higher importance than virtues (e.g., altruism; Kim and Choi, 2015). In Japan, rectitude was considered the most fundamental virtue (Nishigori et al., 2014) whereas in China benevolence and tolerance were important (Jing et al., 2013). Countries with a depressed economy emphasize a good understanding of medical ethics even more due to their prevailing economic situation, limited options of treatment, and cultural setting (Chukwuneke, 2015). Physicians' different religions might also imply different (weighted) virtues, having consequently differing implications for treatment (e.g., Peteet, 2014; Gray, 2017).

Certain roles inherent to the medical profession (e.g., medical specialties, patient clientele) can 'require' certain virtues. Generally, in hospitals, physicians should be team players fulfilling all requirements for motivated and efficient employees (McDougall, 2013). For example in psychiatry, beneficence often conflicts with patients' autonomy or needs (Kwok et al., 2012), where self-effacement could be particularly relevant in the case of prosecuting assaultive patients (Ho et al., 2009). When caring for so-called 'difficult' patients, again the virtues of courage and compassion were emphasized (Hawking et al., 2017). Beside technical skills, surgeons should cultivate practical wisdom (Hall, 2011) and humility (Toledo-Pereyra, 2007), and internists their integrity, respect, and compassion (Bergsma and Thomasa, 1985). Anesthesiologists are often confronted with pain and decision-making or palliative care, so they could particularly benefit from, for instance, justice, temperance, self-effacement, and wisdom according to literature (Diesfeld, 2008; Braun et al., 2010; Guevara-López et al., 2015; Kaldjian, 2019). However, today's culture of medicine (example of the United States) is often hostile to 'truthful' professionalism and other qualities producing 'good' virtuous physicians as medicine has evolved into a giant, increasingly expensive technological profit center with young medical doctors only getting taught a list of required 'professional' practices (Coulehan, 2005).

Relating to character strengths of physicians in terms of the VIA-classification (Peterson and Seligman, 2004), eight empirical studies were identified. Six studies derived from the 'WELLMED' project. Two of the six were using a combination of physicians' and medical students' data (Hausler et al., 2017a; Höfer et al., 2019), three focusing on character strengths' applicability and (work-related) well-being in terms of (a) sociomoral climate (Höge et al., 2019), (b) work characteristics (Strecker et al., 2019), and (c) the distinction of character strengths' application (Huber et al., 2019), and one following a mixed-methods design adding further insights into the relation of character strengths and physicians' well-being (Kachel et al., 2020b). The latter article reports on opinions regarding the most important VIA-character strengths to feel well in the hospital. Resident physicians stated social intelligence, teamwork, perseverance, fairness, and honesty to be most important for well-being at work (descending order), whereas senior educators mentioned the character strength humility to be most relevant, followed by teamwork, kindness, social intelligence, and zest. However, none of these studies focused on identifying a certain physicians' profile. The top five character strengths of the German-speaking hospital physicians from this sample were fairness, honesty, judgment, kindness, and love. Kotzee et al. (2017) asked British established doctors to identify and rank the VIA-character strengths that they think best represent their character and what they think a good doctor would need. There was a strong agreement between physicians and medical 
students concerning fairness, honesty, kindness, perseverance and teamwork representing their character, and that a good doctor is fair, honest, kind, a leader, a good team player, and a person with good judgment. Physicians reported to possess more humor than first-year undergraduates. Finally, in a Swiss physician sample, love of learning was the top character strength, followed by curiosity, creativity, perseverance, perspective, honesty, and social intelligence (Harzer, 2008), with teamwork in the last place. Beside the 'WELL-MED' studies, only data of the latter study revealed physicians' prevalence of specific character strengths by answering the VIA-questionnaire whereas the others originated again from ranking all character strengths by description.

\section{Summary}

Depending on the respective focus, different virtues or character strengths are desirable for medical students and physicians in the literature. The virtues of compassion, courage, altruism, and benevolence were found most often. Summarizing the VIAclassified character strengths, fairness, honesty, kindness, and teamwork were considered most often by respondents to be particularly important among both groups. Finally, according to the Declaration of Geneva, the modern-day physician's pledge states explicitly to respect the patient's autonomy and dignity, despite exercising beneficence and medical confidentiality toward the patients (Parsa-Parsi, 2017). Interestingly, increasing workload, occupational stress, and their potential adverse effects were considered as well in this pledge, leading to the intake of: 'I will attend to my own health, wellbeing, and abilities in order to provide care of the highest standard.' This clause reflects physicians' humanity and their role of self-care being a part in improving patient care, but also offering more possibilities on character building among medical students and physicians due to its positive effect (e.g., Bryan and Babelay, 2009).

\section{Aims and Research Questions}

The literature research revealed a majority of (a) theoretically conceptually driven papers and normative or philosophical research vs. empirical studies, and (b) 'virtues' in general with a striking plurality of different conceptions and theories vs. 'character strengths' in terms of the VIA-classification. Moreover, in previous studies (c) possible character strengths profiles have not been discussed so far also due to the lack of completed VIAquestionnaire data and (d) virtues as well as character strengths were hardly associated with well-being of medical students or physicians themselves but more with the question of ethics in patient care. Therefore, this study aims at adding empirical data concerning VIA-classified character strengths inherent in medical professionals $(\mathrm{a} / \mathrm{b})$ and giving evidence on possible profiles based upon valid questionnaire data with regards to their respective relevance and relation to well-being and work engagement $(\mathrm{c} / \mathrm{d})$. The following three exploratory research questions were addressed:

(I) What character strengths are the most prevalent in a sample of medical students and physicians giving evidence on a possible profile?
(II) Are there any differences in profiles of various sub-groups (e.g., different medical specialties)?

(III) How do character strengths of medical students and physicians relate to well-being and work engagement?

\section{EMPIRICAL DATA}

\section{Methods}

\section{Participants and Procedure}

Data were collected within the 'WELL-MED' project from 2015 to 2020 at an Austrian medical university including two hospitals. In this longitudinal project, person- (e.g., character strengths) and condition-related (e.g., decision latitude, social support, cognitive demands) factors in terms of health and well-being of medical students and hospital physicians were investigated. With institutional review board approval, medical students (human medicine or dentistry) completed an annual online survey over a maximum period of six years, hospital physicians completed three surveys with a time lag of six months. A total of 584 baseline data sets were collected from medical students over the six year period. This sample consisted of 370 women (63.4\%) and 214 men, the mean age was $20.8 \pm 2.5$ years (range: 21 to 38 years), and $55.7 \%$ Austrian, $19.9 \%$ German, and $19.3 \%$ Italian medical students participated. Longitudinal data ( $\mathrm{t} 1$ - t2 - $\mathrm{t} 3$; time lag each one year) were available over a period of three years for 101 medical students. A total of 274 data sets were collected from hospital physicians. About $62 \%$ of them were female $(N=170)$ and the mean age was $34.2 \pm 8.1$ years (range $=24$ to 64 years). A large majority $(N=224 ; 81.8 \%)$ were resident physicians in training, and 50 were senior medical specialists (18.2\%). The physicians worked in 16 different medical disciplines. All participants completed the measurement of character strengths, 217 fully complete data sets were available for $\mathrm{t} 1,90$ for $\mathrm{t} 2$ and 50 for $\mathrm{t} 3$.

\section{Measures}

\section{Character Strengths}

Medical professionals' character strengths were measured with the 'Values in Action - Inventory of Strengths' (VIA-IS; Peterson and Seligman, 2001; Peterson and Park, 2009). Höfer et al. (2019) validated the German short version consisting of 120 -items in total. The 24 character strengths are rated on a five-point Likert scale ranging from 1 (very much unlike me) to 5 (very much like me). VIA-IS mean scores of 3.5 or higher are equal to possessing a character strength at least slightly (Harzer and Ruch, 2013). Item examples are: 'I can always find the positive in what seems negative to others' (hope), 'I never quit a task before it is done' (perseverance), or 'Without exception, I support my teammates or fellow group members' (teamwork). In this sample the internal consistency ranged from $\alpha=0.63$ (teamwork) to $\alpha=0.91$ (spirituality) for medical students, and from $\alpha=0.61$ (teamwork) to $\alpha=0.90$ (spirituality) for physicians.

\section{Well-Being}

General well-being (= thriving) was measured with the German version of the 'Comprehensive Inventory of Thriving' (CIT; 
Hausler et al., 2017). Thriving comprises 18 components, which can be summarized by seven subscales: subjective wellbeing (= SWB; life satisfaction, positive and negative feelings); relationship (support, community, trust, respect, loneliness, belonging), mastery (skills, learning, accomplishment, selfefficacy, self-worth), engagement, autonomy, meaning, and optimism. The latter six can be summarized to psychological well-being (PWB). The 54 items in total are rated on a five-point Likert scale ranging from 1 (strongly disagree) to 5 (strongly agree). Item examples are: 'I am confident that I can deal with unexpected events' (mastery), 'There are people who appreciate me as a person' (relationship), or 'My life has a clear sense of purpose' (meaning). Cronbach's alpha for medical students as well as for physicians in this sample ranged from $\alpha=0.95$ (SWB) to $\alpha=0.92$ (PWB).

\section{Work Engagement}

Work engagement is defined as a fulfilling work-related positive state of mind and characterized by vigor, dedication and absorption (Schaufeli et al., 2006). To measure this construct, the German short version of the 'Utrecht Work Engagement Scale' (UWES; Schaufeli and Bakker, 2003; Schaufeli et al., 2006) was used with one version formulated for students and one for employees. Both consist of nine items, which are rated on a sevenpoint Likert scale ranging from 0 (never) to 6 (always). Item examples are: 'My work inspires me' or 'At my study, I feel strong and vigorous'. Cronbach's alpha was $\alpha=0.94$, for medical students as well as for physicians.

\section{Data Analysis}

For all statistical analyses, SPSS Statistics 26 was used (IBM Corporation, 2018). Pearson's coefficient inter-correlations can be interpreted with $r<0.10=$ no correlation, $r=0.10-0.29=$ low correlation, $r=0.30-0.49=$ moderate correlation, $r \geq 0.50=$ high correlation (Cohen, 1988). Acceptable internal consistency of an instrument is indicated by Cronbach's $\alpha>0.70$ (see Peterson, 1994). T-tests were computed to compare baseline means of two groups (e.g., sex, training status), analyses of variance (ANOVAs) were applied to compare baseline means of multiple groups (e.g., medical specialties). The effect sizes regarding group differences will be represented as Cohen's $d$ ( $>0.2=$ small, $>0.5=$ medium, $>0.8=$ big; Cohen, 1988). Longitudinal regression analyses with all 24 character strengths as predictors were computed with thriving and work engagement as criterion (method: forward; last step mandatory including the criterion variable measured one year or six months before as control variable). Figures of character strengths profiles will not illustrate the whole possible scale spectrum of the VIA-IS (1-5) but a smaller range from 2 to 4.5 to improve readability.

\section{Results}

\section{Medical Students}

\section{(I) Character strengths prevalence}

Among the 584 medical students in this sample (completing t1), the VIA-character strength with the highest reported mean was honesty $(M=4.27, S D=0.47)$, the lowest was spirituality $(M=2.45, S D=1.04)$. Beside honesty, the five highest character strengths mean values ( $M \geq 4.0)$ were found for fairness, judgment, kindness, and love (Table 1). Longitudinal data across three years revealed that these five character strengths remained on top with only little variation suggesting general stability. The order at $\mathrm{t} 2$ was identical, at $\mathrm{t} 3$, honesty and kindness changed the first and second place, and judgment and love the fourth and fifth place. These generally stable positioning trends recurred as well for the subsequent character strengths (e.g., 6th to 10th place). Furthermore, each of the top five character strengths significantly correlated with itself over time (fairness: $r=0.49-0.64$, honesty: $r=0.54-0.58$, judgment: $r=0.69-0.77$, kindness: $r=0.61-0.65$, love: $r=0.61-0.70$; all $p=0.001)$. Figure 1 depicts the character strengths profile for the medical student sample.

\section{(II) Group differences}

Significant differences between female and male medical students were found for 11 character strengths. Women reported higher levels of appreciation of beauty and excellence $(M=3.64$ vs. 3.34; $p<0.001)$, fairness $(M=4.16$ vs. $4.05 ; p<0.05)$, gratitude $(M=3.77$ vs. $3.61 ; p<0.01)$, humility $(M=3.39$ vs. 3.22 ; $p<0.01)$, and love $(M=4.09$ vs. $3.81 ; p<0.001)$; men reported higher levels of bravery $(M=3.47$ vs. $3.69 ; p<0.01)$, creativity $(M=3.33$ vs. $3.47 ; p<0.05)$, humor $(M=3.81$ vs. $3.95 ; p<0.05)$, judgment $(M=4.01$ vs. $4.14 ; p<0.05)$, perspective $(M=3.56$ vs. $3.72 ; p<0.001)$, and self-regulation $(M=3.19$ vs. $3.39 ; p<0.001)$. However, all effect sizes were small (Cohen's $d$ ranging from 0.19 to 0.42 ). Character strength profiles of male and female medical students are displayed in Figure 2.

Medical students' character strengths profiles were also compared regarding the three most desired future medical specialties students wanting to take up. Thus, their profiles were compared for the following groups: trauma and general surgery $(N=95)$, anesthesia, intensive care, and internal medicine $(N=47)$, and pediatric medicine $(N=48)$. According to ANOVA results, significant differences were found for bravery $[F(2,187)=6.99, p=0.001]$, kindness $[F(2,187)=6.72$, $p<0.002]$, and love $[F(2,187)=3.28, p=0.040]$. Medical students being interested in pediatrics had higher mean values concerning kindness $(M=4.54)$ compared to those being interested in internal medicine $(M=4.13, p<0.001, d=0.84)$ or surgery $(M=4.27, p \leq 0.05, d=0.54)$, but they had lower mean values concerning bravery $(M=3.43)$ vs. medical students interested in surgery $(M=3.86 ; p<0.001 ; d=0.66)$. Concerning love, no further significant differences were evident according to the Bonferroni post hoc tests. The character strengths profiles for the three groups are pictured in Figure 3.

\section{(III) Relation to well-being and work-engagement}

The overall mean for thriving was $M=4.01(S D=0.43)$ and for work engagement $M=4.45(S D=0.90)$. Character strengths were positively related to overall well-being (thriving) and mostly to work engagement. Judgment and humility had low or no significant correlations with the well-being subscales, however, spirituality correlated significantly negatively with the subscale 'autonomy'. Forgiveness, humility and spirituality did not significantly correlate with work engagement. In total, the strongest correlations with both outcomes were 
TABLE 1 | The 24 VIA-character strengths of medical students and physicians from the empirical data (t1).

\begin{tabular}{|c|c|c|c|c|c|c|c|}
\hline \multirow[t]{2}{*}{ VIA-character strengths } & Rank & Mean & $S D$ & Min & Max & Skewness & Kurtosis \\
\hline & MS | P & MS | P & MS | P & MS | P & MS | P & MS | P & MS | P \\
\hline Appreciation of Beauty and Excellence & $19 \mid 15$ & $3.53 \mid 3.51$ & $0.74 \mid 0.66$ & $1.0 \mid 1.6$ & $5.0 \mid 5.0$ & $-0.33 \mid-0.11$ & $-0.04 \mid-0.50$ \\
\hline Bravery & $18 \mid 20$ & 3.55 | 3.43 & $0.65 \mid 0.63$ & $1.4 \mid 1.2$ & $5.0 \mid 5.0$ & $-0.08 \mid-0.15$ & $-0.30 \mid 0.01$ \\
\hline Creativity & $21 \mid 18$ & $3.38 \mid 3.45$ & 0.72 | 0.69 & $1.2 \mid 1.0$ & $5.0 \mid 5.0$ & $0.02 \mid-0.25$ & $-0.13 \mid 0.35$ \\
\hline Curiosity & 9 | 8 & 3.84 | 3.84 & $0.60 \mid 0.55$ & $2.0 \mid 2.2$ & $5.0 \mid 5.0$ & $-0.37 \mid-0.37$ & $-0.27 \mid 0.06$ \\
\hline Fairness & $3 \mid 3$ & $4.12 \mid 4.03$ & $0.57 \mid 0.55$ & $1.8 \mid 2.0$ & $5.0 \mid 5.0$ & $-0.61 \mid-0.68$ & 0.52 | 0.61 \\
\hline Forgiveness & $17 \mid 19$ & $3.56 \mid 3.44$ & $0.64 \mid 0.63$ & $1.0 \mid 1.8$ & $5.0 \mid 5.0$ & $-0.22 \mid-0.05$ & $0.01 \mid-0.19$ \\
\hline Gratitude & $11 \mid 14$ & $3.71 \mid 3.53$ & 0.64 | 0.62 & $1.0 \mid 1.8$ & $5.0 \mid 5.0$ & $-0.34 \mid 0.07$ & $0.30 \mid-0.25$ \\
\hline Honesty & $1 \mid 1$ & 4.27 | 4.21 & $0.47 \mid 0.44$ & $2.6 \mid 2.8$ & $5.0 \mid 5.0$ & $-0.48 \mid-0.27$ & $-0.08 \mid-0.19$ \\
\hline Hope & $10 \mid 11$ & $3.80 \mid 3.71$ & $0.68 \mid 0.60$ & $1.4 \mid 2.0$ & $5.0 \mid 5.0$ & $-0.54 \mid-0.31$ & $0.14 \mid-0.01$ \\
\hline Humility & $22 \mid 22$ & $3.33 \mid 3.29$ & $0.67 \mid 0.63$ & $1.6 \mid 1.4$ & $5.0 \mid 4.8$ & $-0.12 \mid-0.10$ & $-0.16 \mid 0.01$ \\
\hline Humor & 8 | 10 & $3.86 \mid 3.71$ & $0.71 \mid 0.68$ & $1.6 \mid 2.0$ & $5.0 \mid 5.0$ & $-0.45 \mid-0.11$ & $-0.14 \mid-0.37$ \\
\hline Judgment & $4 \mid 5$ & 4.05 | 4.00 & $0.60 \mid 0.51$ & $1.4 \mid 2.2$ & $5.0 \mid 5.0$ & $-0.58 \mid-0.20$ & $0.38 \mid-0.09$ \\
\hline Kindness & 2 | 2 & $4.25 \mid 4.10$ & $0.53 \mid 0.50$ & $2.0 \mid 2.8$ & $5.0 \mid 5.0$ & $-0.61 \mid-0.18$ & $0.38 \mid-0.27$ \\
\hline Leadership & 12 | 12 & $3.70 \mid 3.66$ & $0.55 \mid 0.53$ & $1.4 \mid 2.0$ & $5.0 \mid 5.0$ & $0.01 \mid 0.06$ & $0.01 \mid-0.05$ \\
\hline Love & 5 | 4 & $3.99 \mid 4.03$ & $0.67 \mid 0.67$ & $1.2 \mid 1.6$ & $5.0 \mid 5.0$ & $-0.71 \mid-0.82$ & 0.48 | 0.90 \\
\hline Love of Learning & $20 \mid 13$ & $3.51 \mid 3.64$ & $0.74 \mid 0.68$ & $1.6 \mid 1.6$ & $5.0 \mid 5.0$ & $-0.10 \mid-0.09$ & $-0.65 \mid-0.29$ \\
\hline Perseverance & 7 | 6 & $3.88 \mid 3.93$ & $0.65 \mid 0.58$ & $1.8 \mid 2.0$ & $5.0 \mid 5.0$ & $-0.47 \mid-0.55$ & 0.03 | 0.487 \\
\hline Perspective & 15 | 21 & 3.62 | 3.37 & $0.62 \mid 0.53$ & $2.0 \mid 2.0$ & $5.0 \mid 5.0$ & $-0.05 \mid 0.06$ & $-0.40 \mid 0.21$ \\
\hline Prudence & $16 \mid 16$ & 3.57 | 3.49 & 0.65 | 0.60 & $1.4 \mid 1.8$ & $5.0 \mid 4.8$ & $-0.35 \mid-0.16$ & $-0.12 \mid-0.24$ \\
\hline Self-Regulation & $23 \mid 23$ & $3.27 \mid 3.15$ & 0.75 | 0.69 & $1.2 \mid 1.2$ & $5.0 \mid 4.8$ & $-0.04 \mid-0.19$ & $-0.51 \mid-0.14$ \\
\hline Social Intelligence & $6 \mid 7$ & 3.91 | 3.89 & $0.58 \mid 0.52$ & $1.4 \mid 2.4$ & $5.0 \mid 5.0$ & $-0.49 \mid-0.30$ & $0.59 \mid-0.08$ \\
\hline Spirituality & $24 \mid 24$ & $2.45 \mid 2.33$ & 1.04 | 0.95 & $1.0 \mid 1.0$ & $5.0 \mid 5.0$ & 0.54 | 0.56 & $-0.39 \mid-0.32$ \\
\hline Teamwork & 13 | 9 & 3.69 | 3.71 & $0.57 \mid 0.50$ & $1.4 \mid 2.0$ & $5.0 \mid 5.0$ & $-0.28 \mid-0.31$ & 0.52 | 0.54 \\
\hline Zest & $14 \mid 17$ & 3.65 | 3.49 & 0.66 | 0.65 & $1.6 \mid 1.8$ & $5.0 \mid 5.0$ & $-0.31 \mid-0.21$ & $-0.08 \mid-0.25$ \\
\hline
\end{tabular}

Note. MS, medical students $(N=584)$; , physicians $(N=274) ; S D$, Standard deviation.

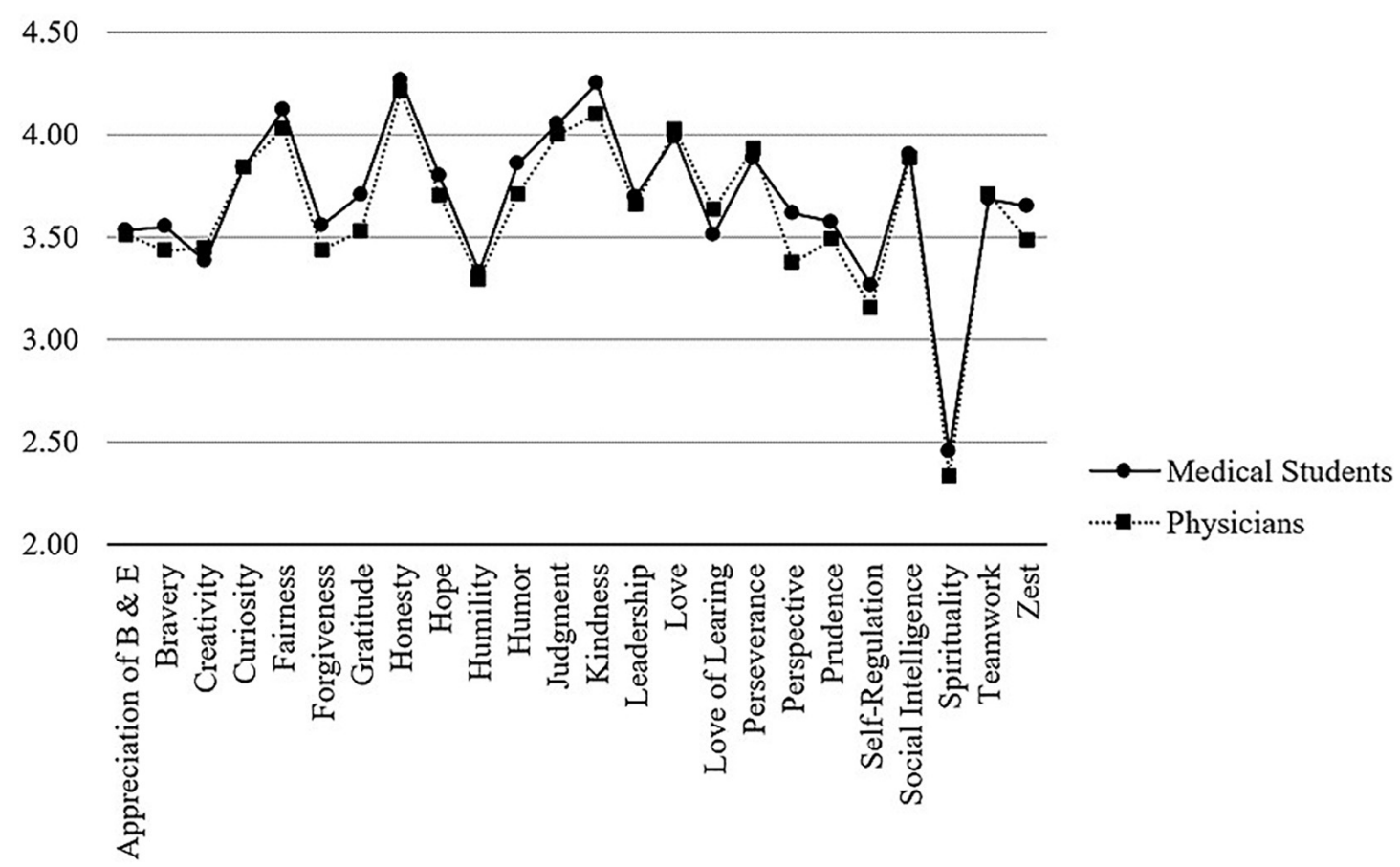

FIGURE 1 | Comparison of VIA-character strengths between medical students and physicians. 

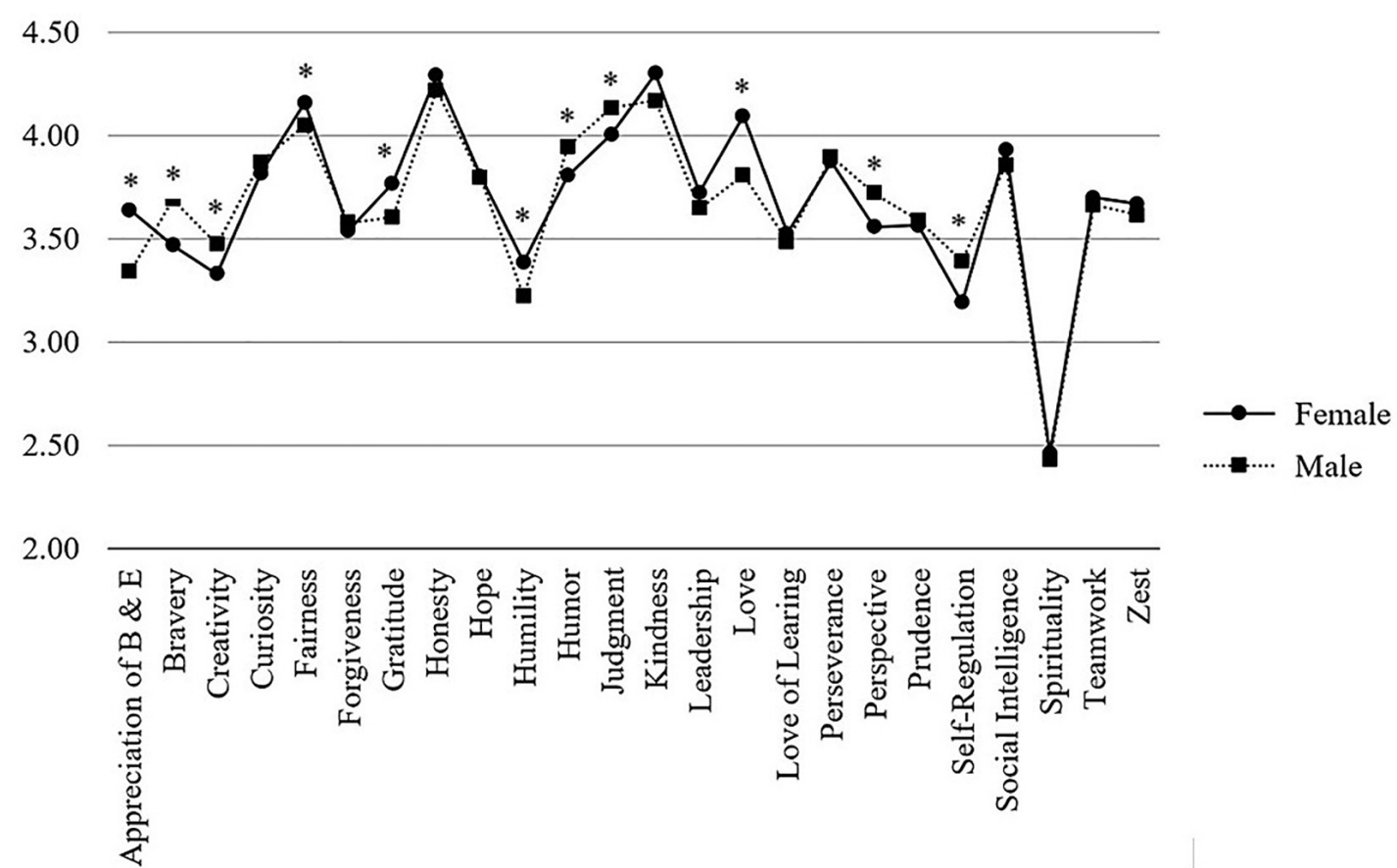

FIGURE 2 | Comparison of VIA-character strengths between male and female medical students. Note. Character strengths marked with an asterisk are significantly different between groups.

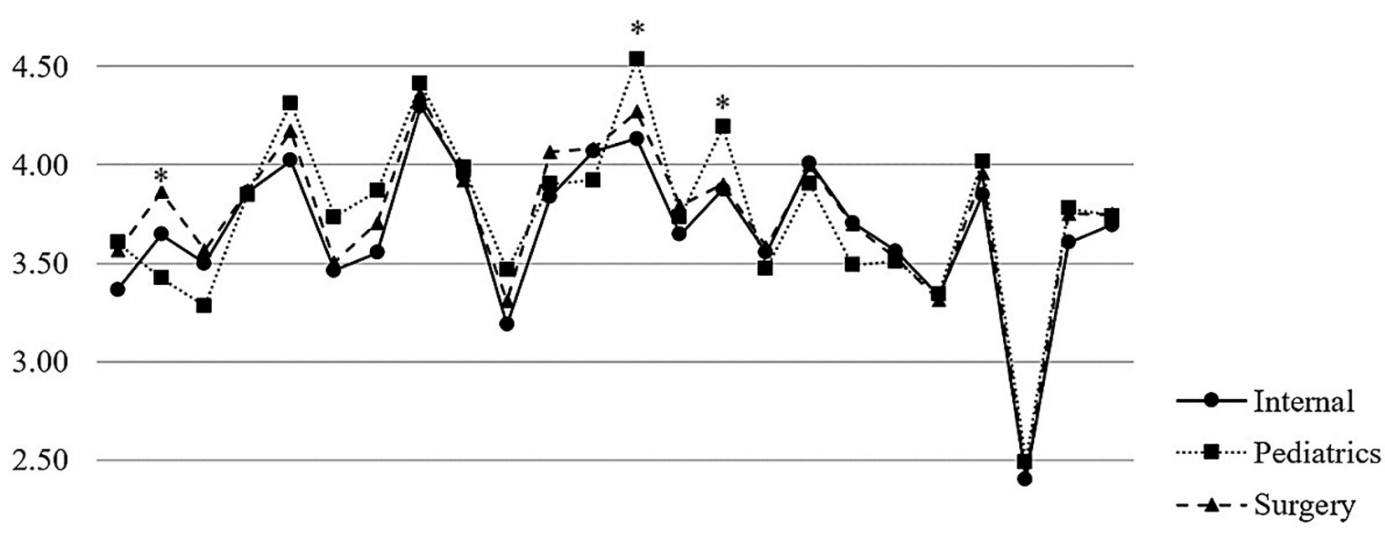

2.00

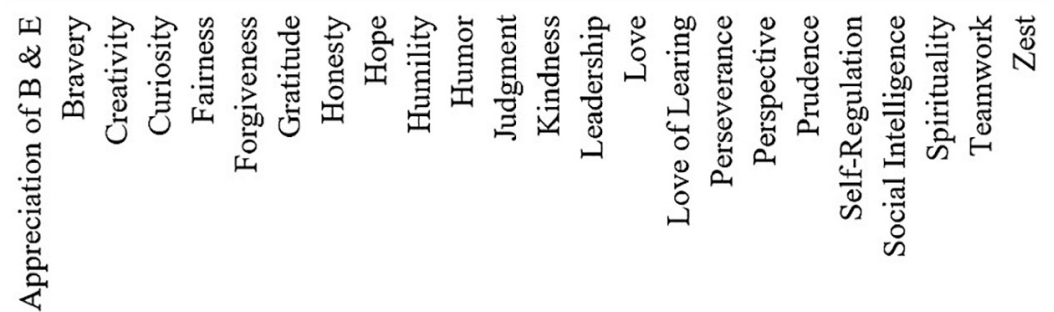

FIGURE 3 | Medical students' VIA-character strengths profile according to the preferred specialty. Note. Character strengths marked with an asterisk are significantly different between groups.

found for the character strengths curiosity, gratitude, hope, love, and zest ('happiness strengths'). All correlation analyses of the VIA-character strengths and thriving with its seven subscales (SWB, relationship, engagement, mastery, autonomy, meaning, and optimism) as well as work engagement are shown in Table 2. 
TABLE 2 | Medical students' correlations between VIA-character strengths and thriving with its seven subscales and work engagement (t1).

\begin{tabular}{|c|c|c|c|c|c|c|c|c|c|}
\hline \multirow[t]{2}{*}{ VIA-character strengths } & \multicolumn{7}{|c|}{ CIT Categories } & \multirow{2}{*}{$\begin{array}{l}\text { Thriving (general } \\
\text { well-being) }\end{array}$} & \multirow{2}{*}{$\begin{array}{c}\text { Work } \\
\text { Engagement }\end{array}$} \\
\hline & Relationship & Engagement & Mastery & Autonomy & Meaning & Optimism & SWB & & \\
\hline $\begin{array}{l}\text { Appreciation of Beauty and } \\
\text { Excellence }\end{array}$ & $0.17^{\star \star}$ & $0.17^{\star \star}$ & $0.22^{\star \star}$ & 0.02 & $0.14^{\star \star}$ & $0.16^{\star \star}$ & $0.10^{\star}$ & $0.21^{\star \star}$ & $0.14^{\star \star}$ \\
\hline Bravery & $0.10^{\star}$ & $0.22^{\star \star}$ & $0.34^{\star \star}$ & 0.03 & $0.22^{\star \star}$ & $0.14^{\star \star}$ & $0.12^{\star \star}$ & $0.23^{\star \star}$ & $0.21^{\star \star}$ \\
\hline Creativity & $0.11^{\star *}$ & $0.26^{\star \star}$ & $0.36^{\star \star}$ & -0.03 & $0.11^{*}$ & $0.14^{\star *}$ & $0.11^{*}$ & $0.22^{\star *}$ & $0.30^{\star *}$ \\
\hline Curiosity & $0.33^{\star \star}$ & $0.46^{\star \star}$ & $0.53^{\star \star}$ & $0.10^{*}$ & $0.30^{\star *}$ & $0.37^{\star \star}$ & $0.41^{\star *}$ & $0.50^{\star \star}$ & $0.43^{\star \star}$ \\
\hline Fairness & $0.28^{\star \star}$ & $0.22^{\star \star}$ & $0.32^{\star \star}$ & $0.11^{*}$ & $0.17^{\star \star}$ & $0.19^{\star \star}$ & $0.19^{\star \star}$ & $0.31^{\star \star}$ & $0.15^{\star \star}$ \\
\hline Forgiveness & $0.16^{\star \star}$ & $0.11^{*}$ & $0.17^{\star \star}$ & 0.02 & $0.11^{*}$ & $0.26^{\star \star}$ & $0.18^{\star \star}$ & $0.21^{\star *}$ & 0.06 \\
\hline Gratitude & $0.34^{\star \star}$ & $0.32^{\star \star}$ & $0.39^{\star \star}$ & 0.08 & $0.41^{\star \star}$ & $0.38^{\star \star}$ & $0.39^{\star \star}$ & $0.46^{\star \star}$ & $0.31^{\star \star}$ \\
\hline Honesty & $0.30^{\star \star}$ & $0.25^{\star \star}$ & $0.36^{\star \star}$ & $0.19^{\star \star}$ & $0.23^{\star \star}$ & $0.18^{\star \star}$ & $0.17^{\star \star}$ & $0.34^{\star \star}$ & $0.16^{\star \star}$ \\
\hline Hope & $0.42^{\star \star}$ & $0.40^{\star \star}$ & $0.56^{\star \star}$ & $0.17^{\star \star}$ & $0.58^{\star \star}$ & $0.70^{\star \star}$ & $0.65^{\star \star}$ & $0.68^{\star \star}$ & $0.42^{\star \star}$ \\
\hline Humility & 0.06 & $0.13^{\star \star}$ & $0.11^{*}$ & 0.07 & 0.05 & 0.04 & 0.04 & $0.09^{\star}$ & -0.03 \\
\hline Humor & $0.25^{\star \star}$ & $0.31^{\star \star}$ & $0.35^{\star \star}$ & 0.03 & $0.22^{\star \star}$ & $0.32^{\star \star}$ & $0.34^{\star \star}$ & $0.37^{\star \star}$ & $0.25^{\star \star}$ \\
\hline Judgment & 0.06 & 0.06 & $0.31^{\star \star}$ & $0.09^{*}$ & $0.12^{\star \star}$ & 0.07 & 0.04 & $0.16^{\star \star}$ & $0.21^{\star \star}$ \\
\hline Kindness & $0.32^{\star \star}$ & $0.20^{\star \star}$ & $0.30^{\star \star}$ & $0.13^{\star \star}$ & $0.17^{\star \star}$ & $0.22^{\star *}$ & $0.20^{\star \star}$ & $0.33^{\star \star}$ & $0.19^{\star \star}$ \\
\hline Leadership & $0.29^{\star *}$ & $0.26^{\star \star}$ & $0.37^{\star \star}$ & 0.03 & $0.20^{\star *}$ & $0.19^{* *}$ & $0.20^{\star \star}$ & $0.33^{\star *}$ & $0.23^{\star *}$ \\
\hline Love & $0.43^{\star \star}$ & $0.25^{\star \star}$ & $0.32^{\star \star}$ & $0.13^{\star \star}$ & $0.27^{\star \star}$ & $0.38^{\star \star}$ & $0.43^{\star \star}$ & $0.47^{\star \star}$ & $0.13^{\star \star}$ \\
\hline Love of Learning & $0.09^{\star}$ & $0.20^{\star \star}$ & $0.32^{\star \star}$ & $0.11^{\star}$ & $0.10^{\star}$ & $0.10^{\star}$ & $0.14^{\star \star}$ & $0.21^{\star \star}$ & $0.34^{\star \star}$ \\
\hline Perseverance & $0.24^{\star \star}$ & $0.31^{* *}$ & $0.45^{\star \star}$ & $0.14^{* *}$ & $0.36^{\star \star}$ & $0.26^{\star \star}$ & $0.24^{\star *}$ & $0.39^{\star *}$ & $0.29^{\star \star}$ \\
\hline Perspective & 0.08 & $0.13^{\star \star}$ & $0.38^{\star \star}$ & 0.09 & $0.17^{\star \star}$ & $0.22^{\star \star}$ & $0.17^{\star \star}$ & $0.25^{\star \star}$ & $0.21^{\star \star}$ \\
\hline Prudence & $0.10^{\star}$ & 0.08 & $0.28^{\star \star}$ & $0.11^{\star}$ & $0.17^{\star \star}$ & $0.13^{\star \star}$ & $0.09^{\star}$ & $0.19^{\star \star}$ & $0.15^{\star \star}$ \\
\hline Self-Regulation & $0.16^{\star \star}$ & $0.31^{\star \star}$ & $0.27^{\star \star}$ & $0.09^{\star}$ & $0.18^{\star \star}$ & $0.17^{\star \star}$ & $0.21^{\star \star}$ & $0.26^{\star \star}$ & $0.22^{\star \star}$ \\
\hline Social Intelligence & $0.35^{\star \star}$ & $0.23^{\star \star}$ & $0.36^{\star \star}$ & $0.12^{* \star}$ & $0.24^{\star \star}$ & $0.28^{\star \star}$ & $0.27^{\star \star}$ & $0.39^{\star \star}$ & $0.26^{\star \star}$ \\
\hline Spirituality & $0.21^{\star \star}$ & $0.13^{\star \star}$ & $0.13^{\star \star}$ & $-0.11^{*}$ & $0.25^{\star \star}$ & $0.19^{\star \star}$ & $0.15^{\star \star}$ & $0.20^{\star \star}$ & 0.07 \\
\hline Teamwork & $0.30^{\star \star}$ & $0.23^{\star \star}$ & $0.29^{\star \star}$ & 0.06 & $0.19^{* \star}$ & $0.18^{\star \star}$ & $0.21^{\star \star}$ & $0.32^{\star \star}$ & $0.19^{\star \star}$ \\
\hline Zest & $0.47^{\star \star}$ & $0.56^{\star \star}$ & $0.56^{\star \star}$ & 0.07 & $0.45^{\star \star}$ & $0.53^{\star *}$ & $0.56^{\star \star}$ & $0.64^{\star \star}$ & $0.48^{\star \star}$ \\
\hline
\end{tabular}

${ }^{*} p \leq 0.05,{ }^{* *} p \leq 0.01$

Concerning the longitudinal regression analyses with all 24 character strengths as predictors, and thriving or work engagement as criterion, stepwise (forward) regression analyses revealed the following. Analyses (time lag one year) with thriving (t2) as criterion $(N=200)$ showed significant positive standardized regression effects for curiosity $(\beta=0.24, t=2.63$, $p=0.009)$ and zest $(\beta=0.37, t=4.17, p<0.001)$ on thriving, while negative effects were apparent for appreciation of beauty and excellence $(\beta=-0.24, t=-3.30, p=0.001)$ and perspective ( $\beta=-0.17, t=-2.13, p=0.034)$. When controlled for thriving at $\mathrm{t} 1$ in a second step, appreciation of beauty and excellence $(\beta=-0.18, t=-2.96, p=0.003)$, perspective $(\beta=-0.14$, $t=-2.05, p=0.042)$, and zest $(\beta=0.21, t=2.65, p=0.009)$ remained significant. Regression analyses $(N=110)$ between character strengths ( $\mathrm{t} 2)$ and thriving $(\mathrm{t} 3)$ showed one significant regression coefficient for hope $(\beta=0.34, \mathrm{t}=2.55, p=0.013)$. When controlled for thriving at $t 2$, no regression analysis remained significant.

Defining work engagement (t2) as criterion and character strengths as predictors $(\mathrm{t} 1)$, analyses $(N=202)$ showed a negative significant standardized regression effect for hope $(\beta=-0.17$, $t=-1.98, p=0.049)$ and a positive one for zest $(\beta=0.42$, $t=3.99, p<0.001)$ on work engagement. When controlled for work engagement at $\mathrm{t} 1$ in a second step, creativity $(\beta=-0.18$, $t=-2.28, p=0.024)$, hope $(\beta=-0.29, t=-3.69, p<0.001)$, and zest $(\beta=0.30, t=3.13, p=0.002)$ appeared significant. Regression analyses examining character strengths (t2) and work engagement ( $\mathrm{t} 3 ; N=111)$ revealed a significant effect for selfregulation $(\beta=0.28, t=2.49, p=0.015)$. When controlled for work engagement at $\mathrm{t} 2$, the regression analysis remained significant for self-regulation $(\beta=0.21, t=2.30, p=0.024)$.

\section{Hospital Physicians}

\section{(I) Character strengths prevalence}

The VIA-character strengths profile in the sample of the 274 hospital physicians (completing $\mathrm{t} 1$ ) resulted in the following. The highest mean value was reported for honesty $(M=4.21$, $S D=0.44)$, and the lowest for spirituality $(M=2.33, S D=0.95)$. Beside honesty, the top five character strengths in this sample $(M \geq 4.0)$ were fairness, judgment, kindness, and love (Table 1). Longitudinal data across three years revealed that these five character strengths remained in front as the top five strengths but with some variation. At $\mathrm{t} 2$, love moved one position forward as well as judgment, while fairness dropped slightly. At t3, judgment and kindness changed the fourth and second place compared to t2. These positioning trends recurred as well for the subsequent character strengths (e.g., 6th to 10th place) suggesting overall general stability. Furthermore, each of the top five character 
strengths significantly correlated with itself over time (fairness: $r=0.68-0.82$, honesty: $r=0.62-0.80$, judgment: $r=0.71-0.75$, kindness: $r=0.69-0.82$, love: $r=0.80-0.86$; all $p=0.001$ ). Figure 1 displays the profile for this sample.

\section{(II) Group differences}

Looking at the differences between character strengths profiles of female and male hospital physicians, results showed an overall homogeneous picture (Figure 4). Significant differences appeared for women reporting higher levels of appreciation of beauty and excellence $(M=3.61$ vs. $3.35, p<0.01)$, gratitude $(M=3.60$ vs. $3.43, p<0.05)$, and spirituality $(M=2.48$ vs. $2.10, p<0.01)$. On the other hand, men rated themselves significantly higher in terms of creativity $(M=3.62$ vs. $3.34, p<0.01)$, curiosity $(M=3.94$ vs. $3.78, p<0.05)$, judgment $(M=4.13$ vs. 3.92, $p<0.01)$, perspective $(M=3.53$ vs. $3.28, p<0.001)$, and prudence $(M=3.59$ vs. $3.43, p<0.05)$. However, all effect sizes were small (Cohen's $d$ ranging from 0.27 to 0.49 ).

Physicians' character strengths profiles were also analyzed regarding their training status which also strongly and naturally correlated with age $(r=0.66, p<0.001)$. The overall picture resulted in a quite homogenous one (Figure 5). Nevertheless, physicians in training scored significantly higher in terms of hope $(M=3.75$ vs. $3.50, p<0.01, d=0.45)$, humor $(M=3.79$ vs. 3.36, $p<0.001, d=0.64)$, and zest $(M=3.52$ vs. 3.34, $p<0.05, d=0.29)$, whereas medical specialists scored significantly $(p<0.05)$ higher in leadership $(M=3.83$ vs. 3.62, $d=0.38)$ and love of learning $(M=3.81$ vs. $3.60, d=0.33)$.

Comparing character strengths profiles of different medical disciplines revealed a general tendency toward the same picture for all hospital physicians in this sample. The focus was on medical disciplines comprising 20 participants or more.
The largest group were physicians with a specialization in anesthesiology $(N=50)$, followed by general surgery $(N=23)$, psychiatry $(N=21)$, and internal medicine $(N=20)$. Significant mean differences were found in 10 of the 24 character strengths and were most often evident when comparing general surgery and psychiatry. Those two groups are depicted in Figure 6, whereas the profiles of anesthesiologists and internal medicals (as they are almost identical to the profile of the physicians in total) will not be depicted for better readability. All results refer mainly to aspiring medical specialists.

According to ANOVA results with Bonferroni post hoc analyses, anesthesiologists - compared to general surgeons - had significantly lower mean values of leadership $[F(3,110)=3.08$, $p<0.05 ; M=3.55$ vs. $M=3.92, p<0.05, d=0.52]$ and perspective $[F(3,110)=3.42, p<0.05 ; M=3.23$ vs. $M=3.60$, $p<0.05, d=0.65]$, whereas they had - compared to psychiatrists - significantly higher mean values of honesty $[F(3,110)=5.06$, $p<0.01 ; M=4.24$ vs. $M=3.94, p<0.01, d=0.71]$ and kindness $[F(3,110)=4.75, p<0.01 ; M=4.18$ vs. $M=3.66, p<0.05$, $d=1.13]$. Internal medicals - compared to psychiatrists - reported significant higher mean values of honesty $[F(3,110)=5.06$, $p<0.01 ; M=4.32$ vs. $M=3.94, p<0.01, d=0.95$ ], humility $[F(3,110)=2.81, p<0.05 ; M=3.47$ vs. $M=2.94, p<0.05$, $d=0.95]$ and judgment $[F(3,110)=3.09, p<0.05 ; M=4.25$ vs. $M=3.84, p<0.05, d=0.93]$. General surgeons had - compared to psychiatrists - significantly higher mean values of honesty $[F(3$, $110)=5.06, p<0.01 ; M=4.37$ vs. $M=3.94, p<0.01, d=1.02]$, leadership $[F(3,110)=3.08, p<0.05 ; M=3.92$ vs. $M=3.47$, $p<0.05, d=0.82]$, prudence $[F(3,110)=3.49, p<0.05 ; M=3.62$ vs. $M=3.11, p<0.05, d=1.19]$, perseverance $[F(3,110)=2.89$, $p<0.05 ; M=4.00$ vs. $M=3.54, p=0.05, d=0.88]$, and zest $[F(3$, $110)=3.24, p<0.05 ; M=3.67$ vs. $M=3.12, p<0.05, d=0.86]$.

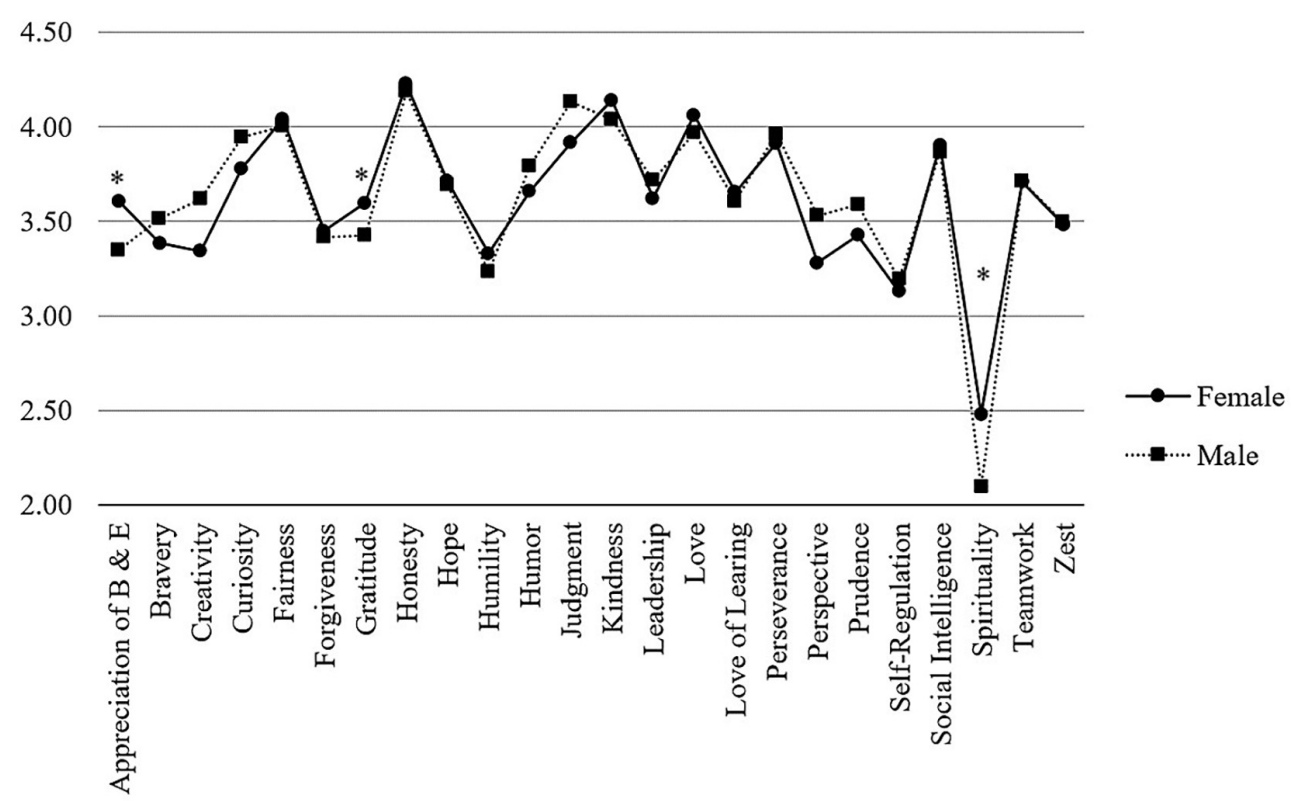

FIGURE 4 | Comparison of VIA-character strengths between male and female physicians. Note. Character strengths marked with an asterisk are significantly different between groups. 


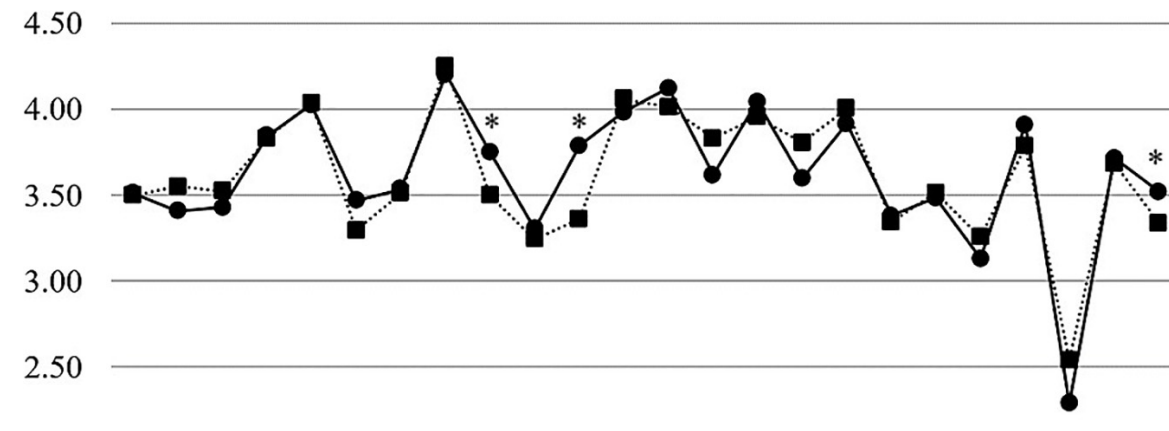

2.00

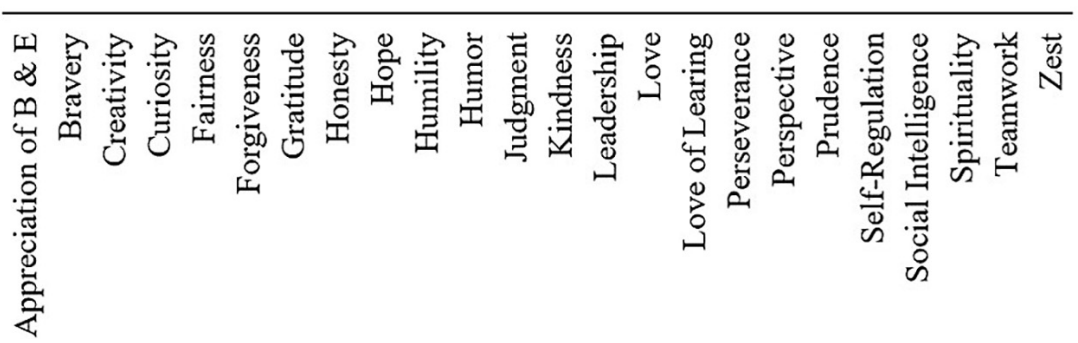

\section{$\rightarrow$ In Training}

-..... Medical Specialists

FIGURE 5 | Comparison of VIA-character strengths between physicians in training and medical specialists. Note. Character strengths marked with an asterisk are significantly different between groups.

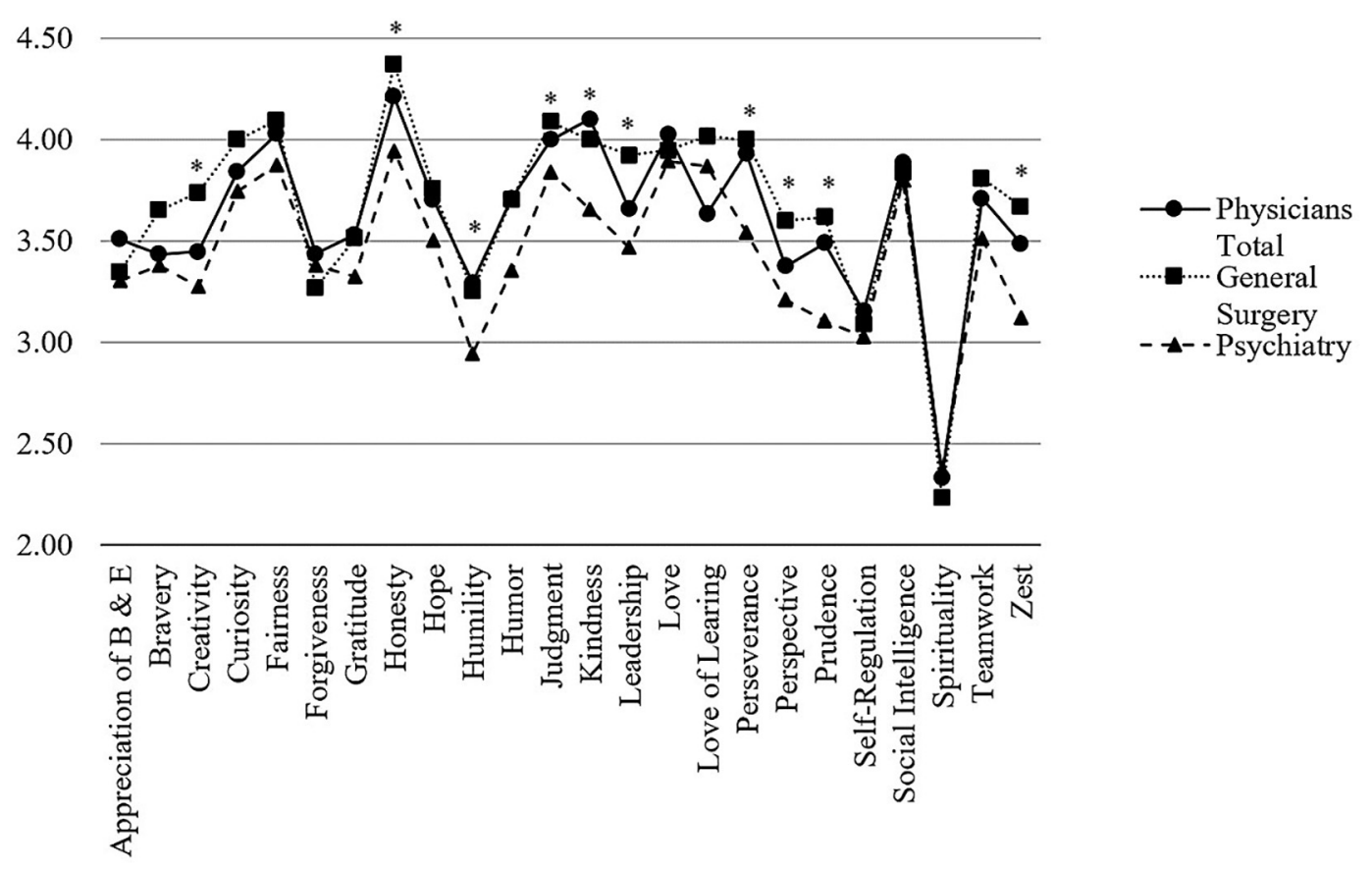

FIGURE 6 | Comparison of VIA-character strengths between physicians of different specialties. Note. Character strengths marked with an asterisk are significantly different between groups.

All differences between medical specialties can be found in the Supplementary Material.

\section{(III) Relation to well-being and work-engagement}

The overall mean for thriving was $M=3.85(S D=0.41)$ and for work engagement $M=3.62(S D=1.08)$. Mostly, character strengths were positively related to overall wellbeing (thriving) and work engagement. Humility and spirituality showed no significant correlations with the well-being subscales, and spirituality was significantly negatively correlated with the subscale 'autonomy'. Appreciation of beauty, fairness, forgiveness, humility, kindness, and spirituality did not significantly correlate 
with work engagement. Again, the 'happiness strengths' showed highest correlations to most well-being subscales, while work engagement correlated the most with curiosity, hope, love of learning, and zest. All correlation analyses of the VIAcharacter strengths and thriving with its seven subscales (SWB, relationship, engagement, mastery, autonomy, meaning, and optimism) as well as work engagement are shown in Table 3.

Concerning the longitudinal regression analyses with all 24 character strengths as predictors, and thriving or work engagement as criterion, stepwise (forward) regression analyses revealed the following. Analyses (time lag 6 months) with thriving (t2) as criterion $(N=88)$ showed significant standardized regression effects for hope $(\beta=0.64, t=8.18, p<0.001)$ and love $(\beta=0.21, t=2.66, p<0.01)$ on thriving. When controlled for thriving at $\mathrm{t} 1$ in a second step, hope $(\beta=0.24, t=2.50, p<0.05)$ remained significant. Regression analyses $(N=50)$ between character strengths (t2) and thriving (t3) showed one significant regression coefficient for hope $(\beta=0.69, t=6.55, p<0.001)$, but when controlled for thriving at $\mathrm{t} 2$, no significant path remained.

Defining work engagement ( $\mathrm{t} 2)$ as criterion and character strengths as predictors $(\mathrm{t} 1)$, analyses $(N=90)$ showed significant standardized regression effects over six months for teamwork $(\beta=0.19, t=2.12, p<0.05)$ and zest $(\beta=0.52, t=5.90$, $p<0.001$ ) on work engagement. When controlled for work engagement at $\mathrm{t} 1$ in a second step, only the control variable remained significant. Performing the same analyses between $\mathrm{t} 2$ and t3 $(N=50)$, significant standardized regression effects over six months revealed for perseverance $(\beta=0.29, t=2.57, p<0.05)$, zest $(\beta=0.55, t=5.06, p<0.001)$, and a negative effect for bravery $(\beta=-0.23, t=-2.10, p<0.05)$ on work engagement. When controlling for work engagement at $\mathrm{t} 2$, a significant path for zest $(\beta=0.34, t=2.06, p<0.05)$ remained while the control variable was not significant for the first time.

\section{DISCUSSION}

This paper reported on known evidence of virtues and VIA-character strengths for medical students and physicians. Literature showed that depending on the respective focus or research area, different virtues and character strengths were evident. Based on the few empirical studies using the VIAclassification of character strengths (Peterson and Seligman, 2004), fairness, honesty, judgment, kindness, and love were reported to have the highest means in medical professionals, even though these results have to be attributed predominantly to the 'WELL-MED' studies. Honesty, fairness, and kindness together with teamwork were consistently rated by medical professionals to be important for being a good doctor. In this study, differences between medical specialties revealed the biggest effect sizes, with psychiatrists consistently reporting lower character

TABLE 3 | Physicians' correlations between VIA-character strengths and thriving with its seven subscales and work engagement (t1).

\begin{tabular}{|c|c|c|c|c|c|c|c|c|c|}
\hline \multirow[t]{2}{*}{ VIA-character strengths } & \multicolumn{7}{|c|}{ CIT Categories } & \multirow{2}{*}{$\begin{array}{c}\text { Thriving } \\
\text { (general } \\
\text { well-being) }\end{array}$} & \multirow{2}{*}{$\begin{array}{c}\text { Work } \\
\text { Engagement }\end{array}$} \\
\hline & Relationship & Engagement & Mastery & Autonomy & Meaning & Optimism & SWB & & \\
\hline Appreciation of Beauty and Excellence & $0.17^{\star}$ & $0.19^{\star *}$ & 0.13 & -0.09 & 0.12 & $0.14^{*}$ & $0.15^{\star}$ & $0.17^{\star \star}$ & 0.06 \\
\hline Bravery & 0.08 & $0.28^{\star \star}$ & $0.23^{\star \star}$ & 0.02 & $0.14^{*}$ & 0.03 & -0.02 & 0.13 & $0.19^{\star \star}$ \\
\hline Creativity & 0.06 & $0.28^{\star \star}$ & $0.34^{\star \star}$ & 0.04 & $0.15^{\star}$ & 0.04 & 0.01 & $0.17^{\star}$ & $0.21^{\star \star}$ \\
\hline Curiosity & $0.28^{\star \star}$ & $0.52^{\star \star}$ & $0.45^{\star \star}$ & $0.18^{\star \star}$ & $0.34^{\star \star}$ & $0.45^{\star \star}$ & $0.38^{\star \star}$ & $0.48^{\star \star}$ & $0.35^{\star \star}$ \\
\hline Fairness & $0.25^{\star \star}$ & $0.27^{\star \star}$ & 0.11 & 0.04 & $0.18^{\star \star}$ & $0.14^{\star}$ & $0.16^{\star}$ & $0.22^{\star \star}$ & 0.10 \\
\hline Forgiveness & $0.20^{\star \star}$ & $0.20^{\star \star}$ & $0.14^{\star}$ & 0.10 & 0.09 & $0.19^{\star \star}$ & $0.18^{\star \star}$ & $0.22^{\star \star}$ & 0.10 \\
\hline Gratitude & $0.40^{\star \star}$ & $0.29^{\star \star}$ & $0.23^{\star \star}$ & 0.00 & $0.31^{\star \star}$ & $0.28^{\star \star}$ & $0.31^{\star \star}$ & $0.38^{\star \star}$ & $0.19^{\star \star}$ \\
\hline Honesty & $0.32^{\star \star}$ & $0.25^{\star \star}$ & $0.23^{\star \star}$ & 0.13 & $0.31^{\star \star}$ & $0.15^{\star}$ & $0.24^{\star \star}$ & $0.33^{\star \star}$ & $0.14^{\star}$ \\
\hline Hope & $0.40^{\star \star}$ & $0.58^{\star \star}$ & $0.55^{\star \star}$ & $0.24^{\star \star}$ & $0.57^{\star \star}$ & $0.64^{\star \star}$ & $0.63^{\star \star}$ & $0.67^{\star \star}$ & $0.48^{\star \star}$ \\
\hline Humility & 0.10 & -0.08 & 0.05 & -0.07 & 0.09 & -0.10 & -0.05 & 0.02 & 0.03 \\
\hline Humor & 0.12 & $0.27^{\star \star}$ & $0.28^{\star \star}$ & -0.02 & 0.10 & $0.31^{\star \star}$ & $0.23^{\star \star}$ & $0.25^{\star \star}$ & $0.24^{\star \star}$ \\
\hline Judgment & 0.06 & $0.18^{\star \star}$ & $0.28^{\star *}$ & 0.12 & 0.08 & 0.03 & 0.06 & $0.16^{\star}$ & $0.14^{*}$ \\
\hline Kindness & $0.26^{\star \star}$ & $0.24^{\star \star}$ & $0.20^{\star \star}$ & -0.05 & $0.15^{\star}$ & $0.17^{\star}$ & $0.15^{\star}$ & $0.24^{\star \star}$ & 0.13 \\
\hline Leadership & $0.15^{\star \star}$ & $0.19^{\star \star}$ & $0.28^{\star \star}$ & 0.04 & $0.23^{\star \star}$ & 0.12 & 0.08 & $0.21^{\star \star}$ & $0.15^{\star}$ \\
\hline Love & $0.49^{\star \star}$ & $0.30^{\star \star}$ & $0.31^{\star \star}$ & $0.16^{\star}$ & $0.39^{\star \star}$ & $0.36^{\star \star}$ & $0.42^{\star \star}$ & $0.50^{\star \star}$ & $0.17^{\star}$ \\
\hline Love of Learning & $0.14^{*}$ & $0.38^{\star \star}$ & $0.30^{\star \star}$ & $0.15^{\star}$ & $0.21^{\star \star}$ & $0.20^{\star *}$ & $0.21^{\star \star}$ & $0.28^{\star \star}$ & $0.33^{\star \star}$ \\
\hline Perseverance & $0.21^{\star \star}$ & $0.28^{\star \star}$ & $0.27^{\star \star}$ & $0.18^{\star \star}$ & $0.34^{\star \star}$ & 0.12 & $0.17^{\star}$ & $0.29^{\star \star}$ & $0.20^{\star \star}$ \\
\hline Perspective & 0.09 & $0.25^{\star \star}$ & $0.34^{\star \star}$ & -0.07 & $0.15^{\star}$ & $0.20^{\star \star}$ & $0.16^{\star}$ & $0.23^{\star \star}$ & $0.26^{\star \star}$ \\
\hline Prudence & $0.15^{\star}$ & 0.12 & $0.23^{\star \star}$ & 0.09 & $0.19^{\star \star}$ & 0.10 & 0.13 & $0.21^{\star \star}$ & $0.19^{\star \star}$ \\
\hline Self-Regulation & $0.16^{\star}$ & 0.14 & 0.12 & 0.00 & $0.17^{\star}$ & 0.09 & 0.13 & $0.17^{\star}$ & $0.15^{\star}$ \\
\hline Social Intelligence & $0.19^{\star \star}$ & $0.24^{\star \star}$ & $0.28^{\star \star}$ & 0.02 & $0.15^{\star \star}$ & $0.23^{\star \star}$ & $0.16^{\star}$ & $0.25^{\star \star}$ & $0.17^{\star}$ \\
\hline Spirituality & $0.15^{\star}$ & -0.07 & -0.04 & $-0.21^{\star \star}$ & 0.05 & -0.06 & -0.03 & 0.01 & -0.01 \\
\hline Teamwork & $0.29^{\star \star}$ & $0.26^{\star \star}$ & $0.28^{\star \star}$ & 0.10 & $0.26^{\star \star}$ & $0.21^{\star \star}$ & $0.24^{\star \star}$ & $0.33^{\star \star}$ & $0.22^{\star \star}$ \\
\hline Zest & $0.40^{\star \star}$ & $0.65^{\star \star}$ & $0.52^{\star \star}$ & $0.17^{*}$ & $0.48^{\star \star}$ & $0.60^{\star \star}$ & $0.59^{\star \star}$ & $0.64^{\star \star}$ & $0.67^{\star \star}$ \\
\hline
\end{tabular}

${ }^{*} p \leq 0.05,{ }^{* *} p \leq 0.01$. 
strength means. The 'happiness strengths' curiosity, gratitude, hope, love, and zest consistently had the highest correlations with thriving cross-sectionally. Long-term results of character strengths influencing well-being and work engagement revealed positive effects (perseverance, self-regulation, teamwork, zest), negative effects (appreciation of beauty and excellence, bravery, creativity, perspective) or even both (hope).

Addressing the first two research questions (I and II), medical students and physicians differed only a little in terms of (1) sex and (2) training status, whereas considerable differences were found regarding their (3) aspired medical specialty.

(1) Female medical students and physicians reported significantly higher values of appreciation of beauty and excellence. Persons with this character strength notice and appreciate beauty, excellence and/or skilled performance in all domains of life, from nature to art to mathematics to science to everyday experience (Seligman, 2002). Other studies found women in general to be more amenable to a conscious perception of beautiful things valuing them (e.g., Ovejero and Cardenal, 2011; Littman-Ovadia and Lavy, 2012). Higher scores regarding love (medical students) and gratitude (both samples) were also shown for women in the two cited studies before, whereas men reported on more creativity (cf. Linley et al., 2007), judgment, and perspective in both samples. Masculine norms (e.g., primacy of work, or pursuit of status) seem to be particularly contrary to appreciation of beauty and excellence beside the fact that men in general reported lower character strengths scores while they tended to increase with comfort to feminine norms (Ovejero and Cardenal, 2011). Socialization processes and gender roles determine the degree to which women and men prioritize morality and experience morally relevant emotions (Ward and King, 2018). Women are expected to be caring and warm (in line with the caregiving role), to consider morality and kindness as integral parts, and to experience negative emotions when people violate the community's welfare. This imprint might also explain the higher means in terms of VIA-character strengths being morally valued traits. However, all effect sizes were consistently small.

(2) Physicians in training reported in particular significantly higher values of humor compared to medical specialists. In the VIA-definition, the scope of humor is intentionally restricted only to forms that serve some moral good, e.g., offering the lighter side to others, making others smile or laugh, building social bonds and lubricating social interaction, or coping with stressful situations. But also other forms of humor exist with some of them being aggressive, self-defeating or clearly mean (e.g., mockery, ridicule, sarcasm) or at the border (e.g., parody, practical jokes; Müller and Ruch, 2011). Possibly, this 'socially warm' humor style pictured by the VIA-IS, is more prevalent in physicians in training as there is more support, solidarity, and collaboration among them and colleagues building social bonds, whereas medical specialists perceive more competition and work mostly on their own. Thus, physicians in training have more possibilities to cultivate relationships with others. Working over years in a hierarchical system with high strain and decreasing valuable social interactions could possibly lead to an increase or change toward other forms of 'humor' not represented in the VIA-IS, e.g., sarcasm or cynicism. Contrarily, one cited study showed that British doctors (with at least five years of experience) reported to possess more humor than medical students (Kotzee et al., 2017). However, when taking a closer look at the sample, only one fifth of them were hospital physicians (vs. general and other private practitioners) possibly supporting the aforementioned assumption that hospitals could re-weigh individuals' character strength toward a different type of humor.

(3) Medical students being interested in pediatrics reported significantly higher means regarding kindness than students interested in surgery or internal medicine. This result makes sense as working with children particularly requires the ability to be caring, supportive, and compassionate with a deep concern for the little patients' welfare. Students being interested in surgery reported more bravery than ongoing pediatrists. This result is consistent with two cited studies where neuroticism (i.e., inhibition, shyness, emotional lability) negatively predicted bravery and surgery was considered to be a more 'realistic' discipline (Petrides and McManus, 2004; Macdonald et al., 2008), implying that surgeons tackle problems, face medical challenges, and react quickly considering immediate consequences. In general, (aspiring) surgeons in this sample reported throughout the highest VIA-means across many character strengths, in particular compared to psychiatrists who rated themselves continuously lower among all character strengths.

Today's medical culture teaches young physicians to develop self-confidence quickly and to move beyond all insecurities. Surgeons might be affected by this issue in particular, as they first have to cause the patient some harm to achieve a benefit for them (e.g., trying saving lives). This might sometimes lead to exaggerated levels of self-confidence and reduced self-reflection, illustrated e.g., by a patient's statement who said he could always tell when surgeons enter the room: 'You enter with an air of bravado and arrogance that the medical doctors do not exude' (Angelos, 2017). Surgeons in this sample rated all character strengths comparatively high, including the character strengths of virtue 'wisdom' (creativity, curiosity, judgment, love of learning, perspective) and humility. This raises the question whether the scores were influenced by increased levels of self-confidence or if they are taking up the idea previously described by Hall (2011) and Toledo-Pereyra (2007) to cultivate practical wisdom or humility beside technical skills. The biggest difference compared to psychiatrists was found for prudence (being careful about one's choices, thinking before acting, involving far-sighted and short-term planning) which is also often referred to as practical wisdom. Obviously, surgeons can cause greater physical, observable, and in the worst case lethal harm when their treatment fails (leading to higher means of prudence). In contrast, psychiatrists treat patients with mental, emotional, and behavioral disorders by developing treatment plans, prescribing medication, conducting conversations, and applying therapeutic interventions. They should have strong listening skills, be perceptive, reflective, and able to provide crisis intervention when needed as their patients cannot be 'cured by scalpel.' Therefore, their way of treating patients is fundamentally different based upon a more holistic (e.g., bio-psycho-social) view on persons' health and disease with 
many chronic patients consulting them again and again. This interpretation of their working style is consistent with some cited studies that considered psychiatry to be rather 'artistic' which is in turn positively associated with 'openness to experience' but also with 'neuroticism' (Petrides and McManus, 2004; Duffy et al., 2009; Woods et al., 2016). However, as this discipline is not as straightforward or concrete as surgery, this might mislead to the assumption of less 'impressive' work, receiving further support from the hospital when paged for patients on the somatic wards for only prescribing psychotropics. Their remaining knowledge or treatment repertoire is oftentimes not asked, conveying little appreciation and a poorer reputation. According to literature, psychiatrists' benevolence often conflicts with patients' autonomy and their self-effacement is relevant (Ho et al., 2009; Kwok et al., 2012). All character strengths can be interpreted as beneficial due to their definition and therefore, possibly striking psychiatrists as being generally 'inappropriate' within their work. Moreover, if honesty (i.e., speaking the truth, presenting oneself and one's reactions genuinely to each person) is understood as the 'opposite' of self-effacement implying to show all internal feelings, intentions, and commitments unfiltered even in precarious situations, the big difference concerning honesty compared to all other medical specialties in this sample would be traceable, as therapists (psychiatrists) should be discreet in sharing honest appraisals with the patient (Salzman, 1973). Therefore, taking all these points together, psychiatrists might remain self-effacing in terms of all character strengths and rate them lower.

Addressing the third research question (III), positive effects of character strengths on well-being have already been demonstrated (e.g., Peterson and Park, 2006; Seligman, 2011) but not many studies illuminated what aspects of well-being are influenced. In this study, various aspects of thriving were cross-sectionally analyzed showing that in both samples humility was mostly not associated with any aspect of thriving and neither were judgment (students) and spirituality (physicians). The latter even had a clearly negative relation with 'autonomy' (control) in both samples. Spirituality comprises many different aspects, e.g., life calling, beliefs about the universe, and practices that connect with the transcendent ('sacred') which is blessed, holy, or particularly special (secular or non-secular). It involves the belief that there is a dimension to life beyond human understanding being in contrast to 'autonomy' defined by life decisions on one's own responsibility, belief in one's personal skills, and internal locus of control. In both samples, humor was positively associated with 'optimism,' and perseverance with 'meaning'. In total, 'mastery' (skills, learning, accomplishment, self-efficacy, and self-worth) was clearly linked to most of the character strengths. In both samples, love of learning was explicitly associated with work engagement.

Longitudinal data examining possible effects of character strengths on later well-being and work engagement revealed significant results for (1) medical students appreciation of beauty and excellence, creativity, hope, perspective, self-regulation, and zest, and (2) physicians' bravery, hope, perseverance, and teamwork.

(1) Initial zest led to positive effects on medical students' well-being one year later whereas appreciation of beauty and excellence and perspective seemed to have a negative impact. Zest implies approaching situations fully tilted with excitement and energy, i.e., being enthusiastic despite all the new demands and strains at the beginning of a medical study. On the other hand, particularly in the first year there is neither the time nor the need (or institutional calling) to recognize, experience, and appreciate beauty around one or others' skills, potentially frustrating students who set a high value on this. Moreover, studying medicine is possibly not that 'beautiful' or 'excellent' as the aspired job afterward, leading to well-being decreases. Perspective (i.e., to think in big terms and avoid getting wrapped up in small details when there are bigger issues to consider) follows the same trajectory in terms of frustration as there are far too many small things at the beginning of a medical study to organize requiring full attention while the bigger picture (e.g., finally becoming a physician) has taken a back seat. Regarding future work engagement, initial creativity, hope, and zest were relevant for outcomes after one year, whereas self-regulation was rather important in the third year. Interestingly, initial creativity and hope influenced work engagement negatively. In medical school, everything is thoroughly structured and planned in the first year following a tight schedule. They have to learn physiology, biochemistry, anatomy, etc. where 'creative' ideas or perspectives might be not asked or even obstructively. Furthermore, first year medical students experience much external control by the institution, educators and examinants contrasting with their hope (e.g., confidence that goals can be reached effectively by one's own agency), leading to less self-efficacy and involvement with working tasks. With increasing demands and strains over time, self-regulation gained relevance for third years' work engagement. This character strength is complex (i.e., regulating one's actions, controlling one's emotions and reactions to disappointment or insecurities) but was also associated with higher 'agreeableness' (e.g., Haslam et al., 2004) including the sub-trait of 'compliance' meaning that one does what one is required or expected to do. This is in line with the finding here, as both support the ability to keep a sense of order and progress in life helping to stay involved with 'work'.

(2) Physicians' longitudinal data revealed that hope had positive effects on their future well-being across all measurement time points. Beside the belief that many effective pathways can be devised in order to get to that desired goal, having positive expectations about the future is inherent to hope. This optimistic thinking can be interpreted as part of well-being in terms of optimism (Scheier and Carver, 1985) also included in the CIT. Physicians' future work engagement was clearly predicted by zest across all time points. As their definitions highly overlap in terms of content (both including excitement, dynamics, and energy with approaching tasks not halfheartedly), other character strengths might provide more information, like bravery, perseverance, or teamwork. In particular, at the beginning of a medical career, teamwork seemed relevant for ensuing work engagement, whereas perseverance was more important in the further course to stay engaged, in contrast to bravery, which had a negative impact on work engagement in the third year. In this context, bravery might have been understood as fulfilling the demand to hang on or withstand physicians' adverse 
working conditions. This strategy could possibly be useful for a short period of time in terms of exploiting oneself toward this requirement but then turning into decreased work engagement with just persevering in the circumstances. When remembering the study of Macdonald et al. (2008) it is quite interesting that the Big Five dimension 'neuroticism' negatively predicted bravery and hope, both having an impact here on physicians' future well-being and work engagement. However, as these effects were directed differently, the role of possible underlying 'neuroticism' is not clear needing further evidence (Macdonald et al., 2008).

Summarizing, character strengths profiles differed in parts for medical specialties, in particular for general surgeons and psychiatrists with biggest effects for honesty and prudence. The top five character strengths were not influential on long-term well-being or work engagement of medical professionals, instead hope, perseverance, self-regulation, teamwork or zest showed more influence over time. Possessing these character strengths alone might not be enough to derive well-being benefits. Applying those character strengths to foster deepened positive experience may be more relevant to increase well-being (e.g., Govindji and Linley, 2007; Littman-Ovadia and Steger, 2010; Seligman, 2011; Harzer and Ruch, 2012, 2013). One study supports the assumption that the possession as well as the applicability of signature character strengths at work and in private life is important but to different degrees, also depending on the respective outcome (Huber et al., 2019). However, that study considered cross-sectional data only. Therefore, deriving longterm well-being or work engagement effects might rather require the application of character strengths.

The question arises whether the top five character strengths in this sample are specific for medical professionals or if their profile is similar compared to other German-speaking samples or socially oriented occupational groups. Ruch et al. (2010) validated the German VIA-IS (240 items) in a Swiss general population sample ( $N=1674)$ with curiosity, fairness, kindness, honesty, and love having the highest means. By investigating women over time, Proyer et al. (2011) found curiosity, love of learning, love, kindness, and fairness to be the top five in Switzerland $(N=1087)$. A study validating the German shorter VIA-IS form with 120 items in a representative sample (Höfer et al., 2019), revealed the highest means for honesty, kindness, fairness, perseverance, and love representing the general German population $(N=1073)$. Results from the VIA-240 in the latter study showed fairness, honesty, kindness, curiosity, humor, and judgment in front. Therefore, it seems that the top five character strengths fairness, honesty, kindness, judgment, and love in the sample of the present study are not that specific for medical professionals but perhaps for the German-speaking population in general. Possibly, socialization in German-speaking countries is particularly oriented toward these character strengths (fairness and kindness being evident in all samples) as they are perceived as important for human development and cohabitation. When compared to other social professions, in particular physicians' honesty and kindness in this sample seemed to be more specific. A recent study investigating character strengths and job satisfaction (Heintz and Ruch, 2020) showed that fairness, judgment, and love were practically always within the top five character strengths of nurses, teachers, and social workers according to VIA-means, beside curiosity and love of learning. Only in their sample of nurses, kindness was placed second and honesty fifth ex aequo with judgment. Therefore, this occupational group showed the highest overlap with physicians from this study. Other samples revealed love of learning and social intelligence to be very important among counselors (compared to a normed sample; Allan et al., 2019) as well as educators, teachers, psychologists, and therapists (Ruch, 2014); judgment and love were highly evident in teachers and psychologists. Harzer (2008) found in Swiss samples again fairness and love in teachers and care workers, and judgment in psychologists, therapists and social workers. Curiosity and spirituality were also repeatedly within their top five character strengths. Contrasting the present findings with prior research and deliberations of a cultural basis of character strengths, at least honesty can be interpreted as being a more specific strength for medical professionals.

There is evidence, on the one hand, that character strengths overlap with personality traits or occupational interests and on the other that they add incremental validity (e.g., Park et al., 2004; West, 2006). However, individual interests, abilities or skills differed strongly from the conceptual VIAclassification although it is more comprehensive than trait and value taxonomies. Other classifying structures like the Five-Factor model (McCrae and Costa, 1997) or Holland's RIASEC-model (1997) might help explain underlying patterns of the top five character strengths found in this empirical study. The character strengths fairness, honesty, kindness, and love can be assigned to e.g., interpersonal strengths, and judgment to intellectual strengths, both significantly correlating with the Big Five dimensions 'agreeableness' and 'openness to experience' (= being inventive and curious vs. consistent/cautious), while interpersonal strengths were also associated with 'conscientiousness' ( $=$ being efficient and organized vs. extravagant/careless; Neto et al., 2014). In detail, fairness and kindness were significantly predicted by 'agreeableness' and 'extraversion' (= being outgoing and energetic vs. solitary/reserved), with the first strength also being predicted by 'conscientiousness' (Noronha and Campos, 2018) and the second by 'openness to experience' (Neto et al., 2014). 'Agreeableness' was clearly related to love (Park et al., 2004) as well as honesty, with 'conscientiousness' also being relevant for the latter strength (Macdonald et al., 2008), and judgment was predicted by 'openness to experience' (Neto et al., 2014). Summarizing, 'agreeableness' can be considered to be the best predictor of the top five character strengths. However, this dimension is considered to be a superordinate trait, including sub-traits like altruism, compliance, empathy, flexibility, honesty, patience, or trust. The RIASEC types are associated with preferences for vocational activities but also with aversions and by analyzing the description of these traits one can derive ideas on their relationship with certain character strengths. Proyer et al. (2012) described other-directed strengths (fairness, kindness), temperance strengths (honesty), and transcendence strengths (love) coming from the VIAYouth (Proyer et al., 2012). They showed that 'social' interests were predicted by other-directed and transcendence strengths, whereas temperance strengths were correlated with 'investigative' 
interests. Littman-Ovadia et al. (2013) found the same significant correlations for 'social' interests but also for 'artistic' ones with the respective character strengths, for judgment 'artistic' and 'investigative' interests were relevant but none significantly for honesty. According to their results, 'artistic' and 'social' interests predominate. However, it is important to keep in mind that some character strengths are related to combinations of personality traits or interests and not stand-alone characteristics.

These results might lead to the hypothesis that medical students and resident physicians in this sample tend to be predominantly 'agreeable' while having mostly 'artistic' and 'social' interests. Such people could be described as rather compassionate, cooperative, emotional, friendly, open, and warm. They prefer tasks involving other people and seem to satisfy their needs in helping situations, being in line with literature on the 'social personality' including doing good for others (see Littman-Ovadia et al., 2013) and the physicians' job description.

\section{Limitations and Implications for Future Research}

First, data were self-reported by the participants implying possible bias in terms of distortion effects. In particular, social desirability of certain character strengths might be possible. A former study already showed that especially 'niceness' strengths (fairness, kindness) but also honesty and love significantly correlated with social desirability (Macdonald et al., 2008). This is in conflict with Peterson and Seligman (2004) assertion that the VIA-IS is free of social desirability effects because all items are socially desirable. Therefore, additional character strengths assessments by peer-ratings (e.g., friends, family or colleagues) would be helpful. Another issue might be the limited number of participants in some medical specialties. Comparing their respective profiles can only give suggestions or trends and need further evidence. The generalizability of the results is limited due to homogenous sampling (e.g., one culture, same language, similar organizational structures, working climate, etc.). Finally, it might be possible that, in contrast to the assumption of character strengths having a causal impact on well-being, a reverse causality may be present in the data. In a recent study, preliminary evidence is given that (psychological) well-being has a significant positive effect on the applicability of signature character strengths over time indicating that higher levels of well-being might be mandatory first to have access to one's own signature strengths (Huber et al., 2020). Applicability in this regard refers to i.a. asking whether a character strength is 'used' at work or in private life (Harzer and Ruch, 2013). It has to be considered that the level of character strengths and their applicability are different constructs. However, as the VIA-items also contain behavior to some extent, there is a certain overlap enabling possible reverse causal effects.

Future research should focus on a fit between personal characteristics in a more holistic sense. Task-related and social demands in different medical specialties may also warrant future research. Exploring a persons' narrative, story and other biography processes (see Borges and Savickas, 2002) could further improve the understanding of how medical students and aspiring physicians tick leading to more comprehensive profiles facilitating career decision-making processes, e.g., when knowing that working with other people is a basic interest vs. working with things or data, different sub-disciplines might be recommended. Future research questions could address this issue by e.g., looking at all kinds of demographic factors, and further examining if certain medical specialties can be assigned to more or less people-orientation and if it would be a flaw to be 'other-oriented' within the respective discipline. Another research direction should focus on how character strengths could be integrated reasonably in the medical curriculum and further education alongside teaching knowledge and skills. Particularly the hidden medical curriculum, often being contrary to positively valued virtues and different across medical facilities, represents a big issue. When looking through medical oaths there is an extreme variation further undergirding diversity of the hidden curricula (Greiner and Kaldjan, 2018). They need to be uncovered and questioned by educators as well as trainees. Global research on culturally and job-related biased differences in medical professionals' character strengths profiles would be necessary to positively influence curricula development suitable for respective cultures and adjuvant for (work-related) well-being.

\section{Conclusion}

This study suggests that the character strengths hope, perseverance, self-regulation, teamwork, and zest are most relevant when it comes to fostering medical students' and physicians' well-being and work engagement. However, these were not part of the top five character strengths reported by the medical professionals. Additionally, negative effects of e.g., bravery, creativity, or perspective on well-being and work engagement were discovered. Creating an institutional environment considering these results could be beneficial for medical professionals' future well-being and health (Strecker et al., 2019). According to the modern-day physician's pledge to pay attention to their own health, the recommendation is to promote self-awareness and character building among medical professionals by considering both individual signature character strengths and 'collective' profiles. Moreover, research on character strengths profiles in medical professionals' must also focus on cultural implications with the need for comparing different societies, working cultures, and other parameters of public health systems (e.g., Western vs. Asian culture), focusing on 'medical common ground'.

\section{DATA AVAILABILITY STATEMENT}

The datasets generated for this study are available on request to the corresponding author.

\section{ETHICS STATEMENT}

This study was conducted in accordance with recommendations of 'The Board for Ethical Questions in Science of the University of Innsbruck' including written informed consent from all subjects. All participants gave written informed consent subject to the 
regulations of the Declaration of Helsinki. The protocol was permitted by 'The Board for Ethical Questions in Science of the University of Innsbruck.'

\section{AUTHOR CONTRIBUTIONS}

$\mathrm{AH}, \mathrm{CS}, \mathrm{TH}$, and $\mathrm{SH}$ were substantially involved in planning and conducting the study. AH drafted the article. CS and TK carried out the data analyses and reported them. All authors revised the manuscript critically for important intellectual content, read and approved the submitted version.

\section{FUNDING}

This research was partly funded by the Austrian Science Fund (FWF) under project number P27228-G22 (Principal

\section{REFERENCES}

Allan, B. A., Owens, R. L., and Douglass, R. P. (2019). Character Strengths in Counselors: Relations With Meaningful Work and Burnout. J. Career Assess. 27, 151-166. doi: 10.1177/10690727177 48666

Angelos. (2017). The right choice? Surgeons, confidence, and humility. Available online at: https://www.mdedge.com/surgery/article/130301/practicemanagement/right-choice-surgeons-confidence-and-humility (accessed February 17, 2017)

Angerer, P., and Weigl, M. (2015). Physicians' Psychosocial Work Conditions and Quality of Care: A Literature Review. Profess. Professional. 5:960. doi: 10.7577/ pp.960

Aramesh, K. (2017). Compassion as the reunion of feminine and masculine virtues in medicine. J. Med. Ethics His. Med. 10, 1-6.

Bain, L. E. (2018). Revisiting the need for virtue in medical practice: a reflection upon the teaching of Edmund Pellegrino. Philos. Ethics Humanit. Med. 13, 1-5. doi: 10.1186/s13010-018-0057-0

Begley, A. M. (2008). Guilty but good: defending voluntary active euthanasia from a virtue perspective. Nurs. Ethics 15, 434-445. doi: 10.1177/096973300 8090514

Behrens, K. G., and Fellingham, R. (2014). Great Expectations: Teaching ethics to medical students in South Africa. Dev. World Bioeth. 14, 142-149. doi: $10.1111 /$ dewb.12017

Bergsma, J., and Thomasa, D. C. (1985). The contribution of ethics and psychology to medicine. Soc. Sci. Med. 20, 745-752. doi: 10.1016/0277-9536(85)90 065-6

Bishop, J. P., and Rees, C. E. (2007). Hero or has-been: Is there a future for altruism in medical education? Adv. Health Sci. Educ. Theory Pract. 12, 391-399. doi: 10.1007/s10459-007-9064-4

Borges, N. J., and Savickas, M. L. (2002). Personality and medical specialty choice: a review and integration. J. Career Assess. 10, 362-380. doi: 10.1177/ 10672702010003006

Borges, N. J., Savickas, M. L., and Jones, B. J. (2004). Holland's theory applied to medical specialty choice. J. Career Assess. 12, 188-206. doi: 10.1177/ 1069072703257755

Brainard, A. H., and Brislen, H. C. (2007). Viewpoint: Learning Professionalism: A View from the Trenches. Acad. Med. 82, 1010-1014. doi: 10.1097/01.ACM. 0000285343.95826 .94

Braun, U. K., Ford, M. E., Beyth, R. J., and McCullough, L. B. (2010). The physician's professional role in end-of-life decision-making: Voices of racially and ethnically diverse physicians. Patient Educ. Counsel. 80, 3-9. doi: 10.1016/j. pec.2009.10.018

Brazeau, C. M. L. R., Shanafelt, T. D., Durning, S. J., Massie, F. S., Eacker, A., Moutier, C., et al. (2014). Distress among matriculating medical students
Investigator: Assoc. Prof. PD Dr. SH, Co-Principal Investigator: PD Dr. TH).

\section{ACKNOWLEDGMENTS}

We express our gratitude to Mirjam Brenner, M.Sc., for her comprehensive support concerning the data administration and processing.

\section{SUPPLEMENTARY MATERIAL}

The Supplementary Material for this article can be found online at: https://www.frontiersin.org/articles/10.3389/fpsyg. 2020.566728/full\#supplementary-material

relative to the general population. Acad. Med. J. Assoc. Am. Med. Colleges 89, 1520-1525. doi: 10.1097/ACM.0000000000000482

Brose, L. A., Rye, M. S., Lutz-Zois, C., and Ross, S. R. (2005). Forgiveness and personality traits. Personal. Individ. Differ. 39, 35-46. doi: 10.1016/j.paid.2004. 11.001

Bryan, C. S. (2007). The seven basic virtues in medicine: summing up. J. South Carolina Med. Assoc. 103, 135-137.

Bryan, C. S., and Babelay, A. M. (2009). Building Character: A Model for Reflective Practice. Acad. Med. 84, 1283-1288. doi: 10.1097/ACM.0b013e318 $1 \mathrm{~b} 6 \mathrm{a} 79 \mathrm{c}$

Buschor, C., Proyer, R. T., and Ruch, W. (2013). Self- and peer-rated character strengths: how do they relate to satisfaction with life and orientations to happiness? J. Posit. Psychol. 8, 116-127. doi: 10.1080/17439760.2012.758305

Buyx, A. M., Maxwell, B., Supper, H., and Schöne-Seifert, B. (2008). Medizinethik als Unterrichtsfach - Lernziele und Evaluation [Medical ethics teaching educational objectives and evaluation]. Wiener Klinische Wochenschrift 120, 655-664. doi: 10.1007/s00508-008-1097-z

Campbell, D. P. (1977). Manual for the Strong-Campbell Interest Inventory, 2nd Edn. Stanford, CA: Stanford University Press.

Carey, G. B., Curlin, F. A., and Yoon, J. D. (2015). Medical student opinions on character development in medical education: a national survey. BMC Res. Notes 8:1-6. doi: 10.1186/s13104-015-1434-z

Cassell, J. (2002). Perturbing the system: "Hard science", "soft science", and social science, the anxiety and madness of method. Hum. Organiz. 61, 177-185. doi: 0018-7259/02/020177-9\$1.40/1

Cawley, M. J., Martin, J. E., and Johnson, J. A. (2000). A virtues approach to personality. Personal. Individ. Differ. 28, 997-1013. doi: 10.1016/S01918869(99)00207-X

Chervenak, F. A., and McCullough, L. B. (2004). Neglected ethical dimensions of the professional liability crisis. Am. J. Obstetr. Gynecol. 190, 1198-1200. doi: 10.1016/j.ajog.2003.10.686

Chukwuneke, F. N. (2015). Ethics of palliative care in late-stage cancer management and end-of-life issues in a depressed economy. Nigerian J. Clin. Pract. 18, S15-S19. doi: 10.4103/1119-3077.170824

Cohen, J. (1988). Statistical power analysis for the behavioral sciences. Hillsdale: Erlbaum.

Corcoran, B. C., Brandt, L., Fleming, D. A., and Gu, C. N. (2016). Fidelity to the healing relationship: a medical student's challenge to contemporary bioethics and prescription for medical practice. J. Med. Ethics 42, 224-228. doi: 10.1136/ medethics-2013-101718

Coulehan, J. (2005). Viewpoint: Today's Professionalism: Engaging the Mind but Not the Heart. Acad. Med. 80, 892-898. doi: 10.1097/00001888-20051000000004

Coulehan, J. (2011). 'A gentle and Humane Temper'. Humility in Medicine. Perspect. Biol. Med. 54, 206-216. doi: 10.1353/pbm.2011.0017 
Diesfeld, K. (2008). Interpersonal Issues Between Pain Physician and Patient: Strategies to Reduce Conflict. Pain Med. 9, 1118-1124. doi: 10.1111/j.15264637.2007.00392.x

DuBois, J. M., Kraus, E. M., Mikulec, A. A., Cruz-Flores, S., and Bakanas, E. (2013). A Humble Task: Restoring Virtue in an Age of Conflicted Interests. Acad. Med. 88, 924-928. doi: 10.1097/ACM.0b013e318294fd5b

Duffy, R. D., Borges, N. J., and Hartung, P. J. (2009). Personality, vocational interests, and work values of medical students. J. Career Assess. 17, 189-200. doi: $10.1177 / 1069072708329035$

Dunn, L. B., Iglewicz, A., and Moutier, C. (2008). A conceptual model of medical student well-being: promoting resilience and preventing burnout. Acad. Psychiatr. 32, 44-53. doi: 10.1176/appi.ap.32.1.44

Dyrbye, L. N., Thomas, M. R., Massie, F. S., Power, D. V., Eacker, A., Harper, W., et al. (2008). Burnout and Suicidal Ideation among U.S. medical students. Ann. Internal Med. 149, 334-370. doi: 10.7326/0003-4819-149-5-200809020-00008

Dyrbye, L. N., West, C. P., Satele, D., Boone, S., Tan, L., Sloan, J., et al. (2014). Burnout among U.S. medical students, residents, and early career physicians relative to the general U.S. population. Acad. Med. 89, 443-451. doi: 10.1097/ ACM.0000000000000134

Emmons, R. A., and McCullough, M. E. (2003). Counting blessings versus burdens: An experimental investigation of gratitude and subjective well-being in daily life. J. Personal. Soc. Psychol. 84, 377-389. doi: 10.1037/0022-3514.84.2.377

Fugelli, P. (1999). Courage - the mother of all virtues [Norwegian article]. Tidsskrift for den Norske laegeforening: tidsskrift for praktisk medicin, ny raekke 119, 1143-1145.

Fuks, A., Brawer, J., and Boudreau, J. D. (2012). The Foundation of Physicianship. Perspect. Biol. Med. 55, 114-126. doi: 10.1353/pbm.2012.0002

Gelhaus, P. (2012). The desired moral attitude of the physician: (I) empathy. Med. Health Care Philos. 15, 103-113. doi: 10.1007/s11019-011-9366-4

Gold, K. J., Sen, A., and Schwenk, T. L. (2013). Details on suicide among US physicians: Data from the National Violent Death Reporting System. General Hospit. Psychiatr. 35, 45-49.

Govindji, R., and Linley, P. A. (2007). Strengths use, self-concordance and wellbeing: Implications for Strengths Coaching and Coaching Psychologists. Int. Coaching Psychol. Rev. 2, 143-153.

Gray, A. (2017). Resilience, spirituality, and health. Psyche Geloof 28, 32-40.

Greiner, A. M., and Kaldjan, L. C. (2018). Rethinking medical oaths using the Physician Charter and ethical virtues. Med. Educ. 52, 826-837. doi: 10.1111/ medu. 13581

Guevara-López, U., Altamirano-Bustamante, M. M., and Viesca-Treviño, C. (2015). New frontiers in the future of palliative care: real-world bioethical dilemmas and axiology of clinical practice. BMC Med. Ethics 16:1-11. doi: 10.1186/s12910-015-0003-2

Hafferty, F. W., and Franks, R. (1994). The Hidden Curriculum, Ethics Teaching, and the Structure of Medical Education. Acad. Med. 69, 861-871. doi: 10.1097/ 00001888-199411000-00001

Hall, D. (2011). The Guild of Surgeons as a Tradition of Moral Enquiry. J. Med. Philos. 36, 114-132. doi: 10.1093/jmp/jhr005

Harzer, C. (2008). "Profile verschiedener Berufe [Profiles of different professions]," in Das VIA-IS in der Laufbahnberatung, eds D. Jungo, W. Ruch, and R. Zihlmann (Bern: SDBB Verlag), 40-42.

Harzer, C., and Ruch, W. (2012). When the job is a calling: The role of applying one's signature strengths at work. J. Posit. Psychol. 7, 362-371. doi: 10.1080/ 17439760.2012.702784

Harzer, C., and Ruch, W. (2013). The Application of Signature Character Strengths and Positive Experiences at Work. J. Happiness Stud. 14, 965-983. doi: 10.1007/ s10902-012-9364-0

Haslam, N., Bain, P., and Neal, D. (2004). The Implicit Structure of Positive Characteristics. Personal. Soc. Psychol. Bull. 30, 529-541. doi: 10.1177/ 0146167203261893

Hausler, M., Huber, A., Strecker, C., Brenner, M., Höge, T., and Höfer, S. (2017). Validierung eines Fragebogens zur umfassenden Operationalisierung von Wohlbefinden. Die deutsche Version des Comprehensive Inventory of Thriving (CIT) und die Kurzversion Brief Inventory of Thriving (BIT). [Validation of an extensive new well-being measure: The German version of the Comprehensive Inventory of Thriving (CIT) and the Brief Inventory of Thriving (BIT)]. Diagnostica 63, 219-228. doi: 10.1026/0012-1924/a000174
Hausler, M., Strecker, C., Huber, A., Brenner, M., Höge, T., and Höfer, S. (2017a). Associations between the Application of Signature Character Strengths, Health and Well-being of Health Professionals. Front. Psychol. 8:1307. doi: 10.3389/ fpsyg.2017.01307

Hausler, M., Strecker, C., Huber, A., Brenner, M., Höge, T., and Höfer, S. (2017b). Distinguishing Relational Aspects of Character Strengths with Subjective and Psychological Well-being. Front. Psychol. 8:1159. doi: 10.3389/fpsyg.2017.01159

Hawking, M., Curlin, F. A., and Yoon, J. D. (2017). Medical education. Courage and compassion: Virtues in Caring for So-called "Difficult" Patients. AMA J. Ethics 19, 357-363.

Heintz, S., and Ruch, W. (2020). Character Strengths and Job Satisfaction: Differential Relationships Across Occupational Groups and Adulthood. Appl. Res. Qual. Life 15, 503-527. doi: 10.1007/s11482-018-9691-3

Hershberger, P. J. (2005). Prescribing Happiness: Positive Psychology and Family Medicine. Fam. Med. 37, 630-634.

Ho, J., Ralston, D. C., McCullough, L. B., and Coverdale, J. H. (2009). When Should Psychiatrists Seek Criminal Prosecution of Assaultive Psychiatric Inpatients? Psychiatr. Ser. 60, 1113-1117. doi: 10.1176/ps.2009.60.8.1113

Höfer, S., Hausler, M., Huber, A., Strecker, C., Renn, D., and Höge, T. (2019). Psychometric characteristics of the German Values in Action Inventory of Strengths 120-Item Short Form. Appl. Res. Qual. Life 15, 597-611. doi: 10.1007/ s11482-018-9696-y

Höge, T., Strecker, C., Hausler, M., Huber, A., and Höfer, S. (2019). Perceived Socio-moral Climate and the Applicability of Signature Character Strengths at Work: a Study among Hospital Physicians. Appl. Res. Qual. Life 15, 463-484. doi: 10.1007/s11482-018-9697-x

Holland, J. L. (1997). Making vocational choices: A theory of vocational personalities and work environments. Odessa, FL: Psychological Assessment Resources.

Huber, A., Bair, A., Höge, T., and Höfer, S. (2020). Do more of what makes you happy? Applying signature character strengths regarding medical students' well-being and health over time. Front. Psychol. (submitted).

Huber, A., Strecker, C., Hausler, M., Kachel, T., Höge, T., and Höfer, S. (2019). Possession and Applicability of Signature Character Strengths: What Is Essential for Well-Being, Work Engagement, and Burnout? Appl. Res. Qual. Life 15, 415-436. doi: 10.1007/s11482-018-9699-8

IBM Corporation (2018). IBM SPSS Statistics for Windows, Version 26.0. Armonk, NY: IBM Corp.

Ishak, R., Lederer, S., Perry, R., Ogunyemi, D., Bernstein, C., and Waguih, N. (2013). Burnout in medical students: a systematic review. Clin. Teacher 10, 242-245.

Jackson, E. R., Shanafelt, T. D., Hasan, O., Satele, D. V., and Dyrbye, L. N. (2016). Burnout and Alcohol Abuse/Dependence Among U.S. Medical Students. Acad. Med. J. Assoc. Am. Med. Colleges 91, 1251-1256. doi: 10.1097/ACM. 0000000000001138

Jacobson, L., Hawthorne, K., and Wood, F. (2006). The 'Mensch' factor in general practice: a role to demonstrate professionalism to students. Br. J. General Pract. 56, 976-979.

Jing, W., Otten, H., Sullivan, L., Lovell-Simons, L., Granek-Catarivas, M., and Fitzsche, K. (2013). Improving the doctor-patient relationship in China: The role of Balint groups. Int. J. Psychiatr. Med. 46, 417-427. doi: 10.2190/PM.46.4.g

Jones, D. (2013). What are the character strengths of a good doctor?. Birmingham: University of Birmingham. Available online at: http://www.jubileecentre.ac.uk/ userfiles/jubileecentre/pdf/insight-series/Jones\%20D.pdf

Kachel, T., Huber, A., Strecker, C., Höge, T., and Höfer, S. (2020a). Development of Cynicism in Medical Students: Exploring the Role of Signature Character Strengths and Well-Being. Front. Psychol. 11, 328. doi: 10.3389/fpsyg.2020. 00328

Kachel, T., Huber, A., Strecker, C., Höge, T., and Höfer, S. (2020b). Reality meets belief: A mixed methods study on character strengths and well-being of hospital physicians. Front. Psychol. (submitted).

Kaldjian, L. (2019). "Wisdom in Medical Decision-Making," in The Cambridge Handbook of Wisdom, eds R. Sternberg and J. Glück (Cambridge: Cambridge University Press), 698-720. doi: 10.1017/9781108568272.032

Karches, K. E., and Sulmasy, D. P. (2016). Justice, Courage, and Truthfulness: Virtues That Medical Trainees Can and Must Learn. Fam. Med. 48, 511-516.

Kim, S., and Choi, S. (2015). The Medical Professionalism of Korean Physicians: Present and Future. BMC Med. Ethics 16:7. doi: 10.1186/s12910-015-0051-7 
Klein, J., Grosse Frie, K., Blum, K., von, and dem Knesebeck, O. (2010). Burnout and perceived quality of care among German clinicians in surgery. Int. J. Qual. Health Care 22, 525-530. doi: 10.1093/intqhc/mzq056

Kotzee, B., Ignatowics, A., and Thomas, H. (2017). Virtue in Medical Practice: An Exploratory Study. HEC Forum 29, 1-19. doi: 10.1007/s10730-016-9308-x

Kwok, S., Matorin, A., and Kahn, D. (2012). Clinical and Ethical Dilemmas in the Psychiatric Care of Patients Who Border on the Edge of Being Able to Live Safely and Independently. J. Psychiatr. Pract. 18, 213-220. doi: 10.1097/01.pra. 0000415079.74238.3f

Larkin, G. L., McKay, M. P., and Angelos, P. (2005). Six core competencies and seven deadly sins: A virtues-based approach to the new guidelines for graduate medical education. Surgery 138, 490-497. doi: 10.1016/j.surg.2005. 03.013

Linley, A., Maltby, J., Wood, A. M., Joseph, S., Harrington, S., Peterson, C., et al. (2007). Character strengths in the United Kingdom: The VIA Inventory of Strengths. Personal. Individ. Differ. 43, 341-351. doi: 10.1016/j.paid.2006. 12.004

Littman-Ovadia, H., and Lavy, S. (2012). Character Strengths in Israel. Hebrew adaptation of the VIA Inventory of Strengths. Eur. J. Psychol. Assess. 28, 41-50. doi: 10.1027/1015-5759/a000089

Littman-Ovadia, H., and Steger, M. F. (2010). Character strengths and wellbeing among volunteers and employees: Toward an integrative model. J. Posit. Psychol. 5, 419-430. doi: 10.1080/17439760.2010.516765

Littman-Ovadia, H., Lavy, S., and Boiman-Meshita, M. (2016). When theory and research collide: examining correlates of signature-strengths use at work. J. Happiness Stud. 18, 527-548. doi: 10.1007/s10902-016-9739-8

Littman-Ovadia, H., Potok, Y., and Ruch, W. (2013). The relationship between vocational personalities and character strengths in adults. Psychology 4, 985993. doi: $10.4236 /$ psych.2013.412142

Livesley, W. J. (2006). “The Dimensional Assessment of Personality Pathology (DAPP) - Approach to Personality Disorder," in Differentiating normal and abnormal personality, ed. S. Strack (New York: Springer Publishing Company), 401-429.

Lopez, L., and Dyck, A. J. (2009). Educating Physicians for moral excellence in the twenty-first century. J. Relig. Ethics 37, 651-668. doi: 10.1111/j.1467-9795.2009. 00406.x

Macdonald, C., Bore, M., and Munro, D. (2008). Values in action scale and the Big 5: An empirical indication of structure. J. Res. Personal. 42, 787-799. doi: 10.1016/j.jrp.2007.10.003

Machado, L., de Oliveira, I. R., Peregrino, A., and Cantilino, A. (2019). Common mental disorders and subjective well-being: Emotional training among medical students based on positive psychology. PLoS One 14:e0211926. doi: 10.1371/ journal.pone.0211926

Magalhães-Sant'Ana, M. (2015). A theoretical framework for human and veterinary medical ethics education. Adv. Health Sci. Educ. 21, 1123-1136. doi: 10.1007/s10459-015-9658-1

Martínez-Martí, M. L., and Ruch, W. (2014). Character strengths and well-being across the life span: data from a representative sample of German-speaking adults living in Switzerland. Front. Psychol. 5:1253. doi: 10.3389/fpsyg.2014. 01253

McCrae, R. R., and Costa, P. T. (1997). Personality trait structure as a human universal. Am. Psychol. 52, 509-516. doi: 10.1037/0003-06 6X.52.5.509

McDougall, R. (2013). Understanding doctors' ethical challenges as role virtue conflicts. Bioethics 27, 20-27. doi: 10.1111/j.1467-8519.2011.01893.x

Miller, G. (2012). Hope Is a Virtue. J. Child Neurol. 27, 1616-1617. doi: 10.1177/ 0883073812459353

Müller, L., and Ruch, W. (2011). Humor and strengths of character. J. Posit. Psychol. 6, 368-376. doi: 10.1080/17439760.2011.592508

Neto, J., Neto, F., and Furnham, A. (2014). Gender and Psychological Correlates of Self-rated Strengths Among Youth. Soc. Indic. Res. 118, 315-327. doi: 10.1007/ s11205-013-0417-5

Nishigori, H., Harrison, R., Busari, J., and Dornan, T. (2014). Bushido and Medical Professionalism in Japan. Acad. Med. 89, 560-563. doi: 10.1097/ACM. 0000000000000176

Noronha, A. P. P., and Campos, R. R. F. (2018). Relationship between character strengths and personality traits. Estudos de Psicologia (Campinas) 35, 29-37. doi: 10.1590/1982-02752018000100004
O'Sullivan, A. J., and Toohey, S. M. (2008). Assessment of professionalism in undergraduate medical students. Med. Teacher 30, 280-286. doi: 10.1080/ 01421590701758640

Olivieri, H. M. (2018). Recta Ratio Agibilium in a medical context: the role of virtue in the physician-patient relationship. Philos. Ethics Humanit. Med. 13, 1-5. doi: 10.1186/s13010-018-0062-3

Otake, K., Shimai, S., Tanaka-Matsumi, J., Otsui, K., and Fredrickson, B. L. (2006). Happy people become happier through kindness: A counting kindnesses intervention. J. Happiness Stud. 7, 361-375. doi: 10.1007/s10902-005-3650-z

Ovejero, M., and Cardenal, V. (2011). "Character strengths with gender perspective. A study with a Spanish sample," in Conference: Second World Congress on Positive Psychology (Philadelphia: Helwan University).

Panambur, S., Al Bandari, M. M. S., and Johari, C. (2017). Desirable Character Strengths of Medical Doctors: Perception of Final Year Medical Students in Oman. Int. J. Sci. Study 5, 289-290. doi: 10.17354/ijss/2017/382

Papadimos, T. J. (2004). Stoicism, the physician, and care of medical outliers. BMC Med. Ethics 5:1-7. doi: 10.1186/1472-6939-5-8

Park, N., Peterson, C., and Seligman, M. P. (2004). Strengths of character and well-being. J. Soc. Clin. Psychol. 23, 603-619. doi: 10.1521/jscp.23.5.603.50748

Parsa-Parsi, R. W. (2017). The Revised Declaration of Geneva. A Modern-Day Physician's Pledge. J. Am. Med. Assoc. 318, 1971-1972. doi: 10.1001/jama.2017. 16230

Pellegrino, E. D. (2002). Professionalism, profession and the virtues of the good physician. Mount Sinai J. Med. 69, 378-384.

Peteet, J. R. (2014). What is the Place of Clinicians' Religious or Spiritual Commitments in Psychotherapy? A Virtues-Based Perspective. J. Rel. Health 53, 1190-1198.

Peterson, C., and Park, N. (2006). Character strengths in organizations. J. Organiz. Behav. 27, 1149-1154. doi: 10.1002/job.398

Peterson, C., and Park, N. (2009). "Classifying and measuring strengths of character," in Oxford handbook of positive psychology, 2nd Edn, eds S. J. Lopez and C. R. Snyder (New York: Oxford University Press), 25-33.

Peterson, C., and Seligman, M. E. P. (2001). Values in Action Inventory of Strengths (VIA-IS). Cincinnati, OH: Values in Action Institute.

Peterson, C., and Seligman, M. E. P. (2004). Character strengths and virtues: A Handbook and Classification. New York: Oxford University Press.

Peterson, R. (1994). A Meta-analysis of Cronbach's Coefficient Alpha. J. Cons. Res. 21, 381-391. doi: 10.1086/209405

Petrides, K. V., and McManus, I. C. (2004). Mapping medical careers: Questionnaire assessment of career preferences in medical school applicants and final-year students. BMC Med. Educ. 4:1-17. doi: 10.1186/1472-6920-4-18

Proyer, R. T., Gander, F., Wyss, T., and Ruch, W. (2011). The relation of character strengths to past, present, and future life satisfaction among German-speaking women. Appl. Psychol. Health Well Being 3, 370-384. doi: 10.1111/j.1758-0854. 2011.01060.x

Proyer, R. T., Sidler, N., Weber, M., and Ruch, W. (2012). A multi-method approach to studying the relationship between character strengths and vocational interests in adolescents. Int. J. Educ. Vocat. Guid. 12, 141-157. doi: 10.1007/ s10775-012-9223-x

Ruch, W. (2014). Charakterstärken: Grundlagen und Anwendungen [Character strengths: background and application]. Switzerland: University of Zurich.

Ruch, W., Proyer, R., Harzer, C., Park, N., Peterson, C., and Seligman, M. E. P. (2010). Values in Action Inventory of Strengths (VIA-IS) Adaptation and Validation of the German Version and the Development of a Peer-Rating Form. J. Individ. Differ. 31, 138-149. doi: 10.1027/1614-0001/a000022

Salzman, L. (1973). Truth, Honesty, and the Therapeutic Process. Am. J. Psychiatr. 130, 1280-1282. doi: 10.1176/ajp.130.11.1280

Sanjai, S., and Gopichandran, V. (2018). Selfless giving in medicine: a study of altruistic attitudes among medical students. Ind. J. Med. Ethics 3, 28-34. doi: 10.20529/IJME.2017.082

Schaufeli, W. B., and Bakker, A. B. (2003). Test manual for the Utrecht Work Engagement Scale. Netherlands: Utrecht University.

Schaufeli, W. B., Bakker, A. B., and Salanova, M. (2006). The measurement of work engagement with a short questionnaire: A cross-national study. Educ. Psychol. Measure. 66, 701-716. doi: 10.1177/0013164405282471 
Scheier, M. F., and Carver, C. S. (1985). Optimism, coping, and health: Assessment and implications of generalized outcome expectancies. Health Psychol. 4, 219247. doi: 10.1037/0278-6133.4.3.219

Schweller, M., Ribeiro, D. L., Celeriz, E. V., and De Carvalho Filho, M. A. (2017). Nurturing virtues of the medical profession: does it enhance medical students' empathy? Int. J. Med. Educ. 8, 262-267. doi: 10.5116/ijme.5951.6044

Sehiralti, M., Akpinar, A., and Ersoy, N. (2010). Attributes of a good physician: what are the opinions of first-year medical students? J. Med. Ethics 36, 121-125. doi: 10.1136/jme.2009.032854

Seligman, M. E. P. (2002). Authentic Happiness: Using the new positive psychology to realize your potential for lasting fulfillment. New York: Free Press.

Seligman, M. E. P. (2011). Flourish: A visionary new understanding of happiness and wellbeing. New York, NY: Free Press.

Seligman, M. E. P., and Csikszentmihalyi, M. (2000). Positive psychology: An introduction. Am. Psychol. 55, 5-14. doi: 10.1037//0003-066X.55.1.5

Sheldon, K. M., and Lyubomirsky, S. (2004). “Achieving sustainable new happiness: Prospects, practices, and prescriptions," in Positive Psychology in practice, eds A. Linley and S. Joseph (Hoboken, NJ: John Wiley \& Sons), 127-145.

Shelp, E. E. (1984). Courage: a neglected virtue in the patient-physician relationship. Soc. Sci. Med. 18, 351-360. doi: 10.1016/0277-9536(84)90125-4

Shepherd, A., Schnitker, S. S., Leffel, G. M., Oakes Mueller, R. A., Curlin, F. A., Yoon, J. D., et al. (2018). Developing the Good Physician: Spirituality affects the development of virtues and moral intuitions in medical students. J. Posit. Psychol. 13, 143-154. doi: 10.1080/17439760.2016.1269185

Strecker, C., Huber, A., Höge, T., Hausler, M., and Höfer, S. (2019). Identifying thriving Workplaces in Hospitals: Work Characteristics and the Applicability of Character Strengths at Work. Appl. Res. Qual. Life 15, 437-461. doi: 10.1007/ s11482-018-9693-1

Toledo-Pereyra, L. H. (2007). Humility. J. Investig. Surg. 20, 63-65. doi: 10.1080/ 08941930701234588
Wallace, J. E., Lemaire, J. B., and Ghali, W. A. (2009). Physician wellness: a missing quality indicator. Lancet 374, 1714-1721. doi: 10.1016/S0140-6736(09)61424-0 Ward, S. J., and King, L. A. (2018). Gender Differences in Emotion Explain Women's Lower Immoral Intentions and Harsher Moral Condemnation. Personal. Soc. Psychol. Bull. 44, 653-669. doi: 10.1177/0146167217744525

Wear, D., and Zarconi, J. (2008). Can Compassion be Taught? Let's Ask Our Students. J. General Internal Med. 23, 948-953. doi: 10.1007/s11606-0070501-0

West, M. N. (2006). The Relationship Among Personality Traits, Character Strengths, and Life Satisfaction in College Students. Doctoral Dissertation, University of Tennessee. Tennessee.

Wood, A. M., Joseph, S., and Maltby, J. (2008). Gratitude predicts psychological well-being above Big Five facets. Personal. Individ. Differ. 46, 443-447. doi: 10.1016/j.paid.2008.11.012

Woods, S. A., Patterson, F. C., Wille, B., and Koczwara, A. (2016). Personality and occupational specialty. An examination of medical specialties using Holland's RIASEC model. Career Dev. Int. 21, 262-278. doi: 10.1108/CDI-10-201 5-0130

Conflict of Interest: The authors declare that the research was conducted in the absence of any commercial or financial relationships that could be construed as a potential conflict of interest.

Copyright (c) 2020 Huber, Strecker, Kachel, Höge and Höfer. This is an open-access article distributed under the terms of the Creative Commons Attribution License (CC BY). The use, distribution or reproduction in other forums is permitted, provided the original author(s) and the copyright owner(s) are credited and that the original publication in this journal is cited, in accordance with accepted academic practice. No use, distribution or reproduction is permitted which does not comply with these terms. 